\title{
Stability analysis of a tritrophic model with stage structure in the prey population
}

\author{
Gamaliel Bléa,*, Miguel Angel Dela-Rosa ${ }^{\mathrm{b}}$, Iván Loreto-Hernández ${ }^{\mathrm{b}}$ \\ ${ }^{a}$ División Académica de Ciencias Básicas, UJAT, Km 1, Carretera Cunduacán-Jalpa de Méndez, Cunduacán, Tabasco, c.p. 86690, \\ México. \\ ${ }^{b}$ División Académica de Ciencias Básicas, CONACyT-UJAT, Km 1, Carretera Cunduacán-Jalpa de Méndez, Cunduacán, Tabasco, c.p. \\ 86690, México.
}

\begin{abstract}
We analyze the role of the age structure of a prey in the dynamics of a tritrophic model. We study the effect of predation on a non-reproductive prey class, when the reproductive class of the prey has a defense mechanism. We consider two cases accordingly to the interaction between predator and reproductive class of the prey. In the first case, the functional response is Holling type II and it is possible to show up to two positive equilibria. When we consider a defense mechanism the functional response is Holling type IV. In both cases, we show sufficient parameter conditions to have a stable limit cycle obtained by a supercritical Hopf bifurcation. Some numerical simulations are carried out.
\end{abstract}

Keywords: Hopf's Bifurcation, tritrophic model, coexistence of species, prey age structure.

2010 MSC: 37G15, 34C23, 92D40, 92D25.

(C)2019 All rights reserved.

\section{Introduction}

The mathematical modelling has become a very useful tool in ecology because it can be used to answer general or specific questions about an ecosystem. One of the interactions between species that has been most studied is the predator-prey type. Its study began with the Lotka-Volterra model, from which other models have been obtained considering more variables and more parameters in order to be closer to reality, [13]. For example, it has been included the carrying capacity, the handling time, the interference among predators and defense mechanism, among others. Since the age specific fecundity or fertility rate of a population is one of the most fundamental parameter in both the theory and practice of populations dynamics, [3], the study of age structured models is a topic of ecological interest.

The analysis of prey-predator interaction with age structure has been approached with different models. At first, the structure of ages was considered in the predator population, such is the case of Beddington et al. work, who studied a difference equations system dividing the predator population into the

\footnotetext{
*Corresponding author

Email addresses: gble@ujat.mx (Gamaliel Blé), madelarosaca@conacyt.mx (Miguel Angel Dela-Rosa), iloretohe@conacyt.mx (Iván Loreto-Hernández)
}

doi: $10.22436 /$ jnsa.012.12.01

Received: 2019-04-05 Revised: 2019-05-31 Accepted: 2019-06-08 
young and adult class and assuming that each one has a different attack rate. They showed that stable coexistence is possible, [1]. Hasting et al. analyzed a differential equations system with age structure in the predator. They proved the existence of stable equilibria and determined the effect of age structure, [6]. Cushing et al. studied an integro differential equations system derived from the McKendrick model. They considered an age structure in the predator and proved the parameter conditions to have the coexistence, [3]. Toth analyzed the effects of age structure on the predator prey resource chemostat model, he proved the coexistence equilibrium and the parameter condition to have a Hopf bifurcation, [19]. Xi et al. analyzed a delayed tritrophic food chain model with stage structure in predator and superpredator populations. They determined sufficient conditions to have positivity and permanence in the solution of the system, [20].

On the other hand, in nature it has also been found predators that eat only adults, or immature prey, it is the cicada case, which is preyed only in adult stage, or some species of perch which feed on immature prey, [9-11]. Hence, it is important to study the model with age structure in the prey population. Zhang et al. considered a predator prey model with two stage structure in the prey (immature and mature). They supposed that predator feeds only on the immature class with Lotka-Volterra functional response and they obtained necessary and sufficient conditions for the coexistence or extinction. Falconi, analyzed a predator prey model, dividing the prey population in the reproductive and non reproductive class. He considered three different functional responses to predation on the nonreproductive class and he showed the conditions to have the coexistence, [5]. More recently, it has been considered the analysis of predator prey systems with age structure in the population prey or in the population predator. Tang et al. analyzed a predator prey model with age structure in the predator population. They showed the existence and uniqueness of positive equilibrium and exhibit a Hopf bifurcation, [18]. Promrak et al. considered a predator prey model with age structure in the prey population. They showed the stability solutions and the bifurcation diagrams of the system, [15].

In this paper we analyze a tritrophic model focusing on two classes (reproductive and nonreproductive) in the prey population. Reproductive population will be denoted as $w$, the non-reproductive by $x$, the predator and superpredator populations by $\mathrm{y}$ and $z$, respectively. We will assume that the predator population attacks in a different way to the two classes in the prey. The non-reproductive class contains the oldest organisms and its interaction with the predator is of a Lotka-Volterra type. The interaction between the reproductive population and predator is modeled by a functional response Holling type II or $\mathrm{IV}$, this last functional response considers a defense mechanism in the reproductive class. Explicitly, we have the following differential equations system

$$
\begin{aligned}
& \frac{d w}{d t}=w \rho\left(1-\frac{w+x}{R}\right)-f_{1}(w, x) y-v w, \quad \frac{d x}{d t}=v w-d_{1} x-a_{3} x y, \\
& \frac{d y}{d t}=c_{1} y f_{1}(w, x)+c_{2} a_{3} x y-d_{2} y-\frac{a_{2} y}{y+b_{2}} z, \quad \frac{d z}{d t}=c_{3} \frac{a_{2} y}{y+b_{2}} z-d_{3} z .
\end{aligned}
$$

We consider two cases, which are obtained by considering that the functional response $f_{1}(w, x)=$ $\frac{a_{1} w}{w+x+b_{1}}$ or $f_{1}(w, x)=\frac{a_{1} w}{w^{2}+x+b_{1}}$. Even though these functional responses are not precisely of the classical Holling type (see for instance [4, 17]), through this paper we will call them of Holling type II and $\mathrm{IV}$, respectively. We assume that the birth rate of the non reproductive population is proportional to the reproductive one with proportionality constant $v$, hence the non reproductive population does not extinguish unless the reproductive class does.

For ecological reasons, all the parameters are positive and we restrict our analysis to the positive set $\Gamma:=\left\{(w, x, y, z) \in \mathbb{R}^{4}: w>0, x>0, y>0, z>0\right\}$.

\section{Criteria for stability and Hopf bifurcation}

In next lemma we characterize under some hypothesis the Routh-Hurwitz test, [16], and the necessary condition to have a Hopf bifurcation. 
Let $\operatorname{pol}(\lambda)=\lambda^{4}+A_{1} \lambda^{3}+A_{2} \lambda^{2}+A_{3} \lambda+A_{4}$ be the characteristic polynomial for the linear approximation of the system (1.1) at some equilibrium point P. Set

$$
\text { EQ }:=A_{1}^{2} A_{4}-A_{1} A_{2} A_{3}+A_{3}^{2} .
$$

On the other hand, to have a Hopf bifurcation of the differential system (1.1) at $\mathrm{P}$ it is needed that the characteristic polynomial $\operatorname{pol}(\lambda)$ at $\mathrm{P}$ factorizes as

$$
\left(\lambda-\lambda_{3}\right)\left(\lambda-\lambda_{4}\right)\left(\lambda^{2}+\omega^{2}\right), \omega>0 .
$$

In this notation we state the following criteria which will be used later.

Lemma 2.1. If $A_{i}>0, i=1, \ldots, 4$, the following statements hold.

(i) The equilibrium point $\mathrm{P}$ is locally asymptotically stable if and only if $\mathbf{E Q}<0$.

(ii) Assume that $\operatorname{pol}(\lambda)$ factorizes as in (2.2). Then, its roots are

$$
\omega= \pm i \sqrt{\frac{A_{3}}{A_{1}}} \text { and } \lambda_{3,4}=\frac{-A_{1} \pm \sqrt{A_{1}^{2}-4 A_{2}+4 \omega^{2}}}{2}
$$

if and only if $\mathbf{E Q}=0$.

Proof. We prove (i). Under the hypothesis, the claim follows directly observing that the Hurwitz determinants for $\operatorname{pol}(\lambda)$ are given by

$$
\operatorname{det}_{1}=A_{1}, \quad \operatorname{det}_{2}=A_{1} A_{2}-A_{3}, \quad \operatorname{det}_{3}=-A_{3}^{2}+A_{1}^{2}\left(-A_{4}\right)+A_{1} A_{2} A_{3}, \operatorname{det}_{4}=-A_{4}\left(-A_{1} A_{2} A_{3}+A_{3}^{2}+A_{1}^{2} A_{4}\right)
$$

and all are positive if and only if $A_{2}>\frac{A_{1}^{2} A_{4}+A_{3}^{2}}{A_{1} A_{3}}$. We now prove (ii). Assume that $\operatorname{pol}(\lambda)$ is as in the hypothesis. Then it factorizes as

$$
P(\lambda)=\left(\lambda-\alpha_{1}\right)\left(\lambda-\alpha_{2}\right)\left(\lambda^{2}+\omega^{2}\right),
$$

where $\omega>0$ if and only if

$$
A_{1}=-\left(\alpha_{1}+\alpha_{2}\right), \quad A_{2}=\left(\omega^{2}+\alpha_{1} \alpha_{2}\right), \quad A_{3}=-\left(\alpha_{1}+\alpha_{2}\right) \omega^{2}, \quad A_{4}=\alpha_{1} \alpha_{2} \omega^{2} .
$$

Equivalently,

$$
A_{2}=\left(\omega^{2}+\alpha_{1} \alpha_{2}\right), \quad A_{3}=A_{1} \omega^{2}, A_{4}=\alpha_{1} \alpha_{2} \omega^{2} .
$$

That is,

$$
\alpha_{1} \alpha_{2}=A_{2}-\omega^{2}, A_{3}=A_{1} \omega^{2}, A_{4}=\alpha_{1} \alpha_{2} \omega^{2} .
$$

Equivalently,

that is,

$$
\left(-A_{1}-\alpha_{2}\right) \alpha_{2}=A_{2}-\frac{A_{3}}{A_{1}} \text { and } A_{4}=\left(A_{2}-\frac{A_{3}}{A_{1}}\right) \frac{A_{3}}{A_{1}}
$$

This completes the proof.

$$
\alpha_{2}^{2}+A_{1} \alpha_{2}+\frac{A_{2} A_{1}-A_{3}}{A_{1}}=0 \text { and } A_{1}^{2} A_{4}-A_{1} A_{2} A_{3}+A_{3}^{2}=0 .
$$

Along this paper, the transversality condition to have a Hopf bifurcation will be computed by means of the following result that appeared as an exercise in [7, p. 189].

Proposition 2.2. Let $\mathrm{M}(\tau)$ be a parameter-dependent real $(\mathrm{n} \times \mathrm{n})$-matrix which has a simple pair of complex eigenvalues $\xi(\tau) \pm i \omega(\tau)$ such that $\xi\left(\tau_{0}\right)=0$ and $\omega\left(\tau_{0}\right):=\omega_{0}>0$. Then, the derivative of the real part of the complex eigenvalues is given by

$$
\frac{\mathrm{d} \xi}{\mathrm{d} \tau}\left(\tau_{0}\right)=\operatorname{Re}\left(\overline{\mathbf{p}}^{\mathrm{tr}} \cdot\left(\frac{\mathrm{d} M}{\mathrm{~d} \tau}\left(\tau_{0}\right) \cdot \mathbf{q}\right)\right),
$$

where $\mathbf{p}, \mathbf{q} \in \mathbb{C}^{n}$ are eigenvectors satisfying the normalization conditions

$$
M\left(\tau_{0}\right) \mathbf{q}=i \omega_{0} \mathbf{q}, \quad M^{\operatorname{tr}}\left(\tau_{0}\right) \mathbf{p}=-i \omega_{0} \mathbf{p}, \quad \text { and } \overline{\mathbf{p}}^{\operatorname{tr}} \cdot \mathbf{q}=1
$$




\section{Case $f_{1}(w, x)$ Holling II}

Lemma 3.1. The differential system (1.1) has:

(i) A unique equilibrium point in $\Gamma$ if

$$
a_{2}=\frac{d_{3}+k_{1}}{c_{3}}, \quad d_{2}=\frac{a_{1} c_{1} k_{1} k_{2}\left(a_{3} b_{2} d_{3}+d_{1} k_{1}\right)}{\left(a_{3} b_{2} d_{3}+k_{1}\left(d_{1}+v\right)\right)\left(a_{1} b_{2} d_{3}+k_{1}\left(b_{1} v+k_{2}\right)\right)}, \quad \rho=\frac{a_{1} b_{2} d_{3}}{b_{1} k_{1}}+v, \quad R=\frac{b_{1}\left(a_{1} b_{2} d_{3}+k_{1}\left(b_{1} v+k_{2}\right)\right)}{a_{1} b_{2} d_{3}},
$$

where $k_{1}$ and $k_{2}$ are positive real numbers. The equilibrium is given by

$$
P_{0}=\left(\frac{b_{1} k_{1} k_{2}\left(a_{3} b_{2} d_{3}+d_{1} k_{1}\right)}{\left(a_{1} b_{2} d_{3}+b_{1} k_{1} v\right)\left(a_{3} b_{2} d_{3}+k_{1}\left(d_{1}+v\right)\right)}, \frac{b_{1} k_{1}^{2} k_{2} v}{\left(a_{1} b_{2} d_{3}+b_{1} k_{1} v\right)\left(a_{3} b_{2} d_{3}+k_{1}\left(d_{1}+v\right)\right)}, \frac{b_{2} d_{3}}{k_{1}}, \frac{a_{3} b_{1} b_{2} c_{2} c_{3} k_{1} k_{2} v}{\left(a_{1} b_{2} d_{3}+b_{1} k_{1} v\right)\left(a_{3} b_{2} d_{3}+k_{1}\left(d_{1}+v\right)\right)}\right) .
$$

(ii) Two equilibrium points in $\Gamma$ if

$$
\begin{aligned}
& a_{1}=k_{2}\left(\frac{k_{2}\left(w_{0}+x_{0}\right)}{4 w_{0} y_{0}\left(2 d_{1} x_{0}+2 k_{1}+k_{2}\right)}+\frac{w_{0}+x_{0}}{2 w_{0} y_{0}}\right), a_{2}=\frac{d_{3}\left(b_{2}+y_{0}\right)}{c_{3} y_{0}}, a_{3}=\frac{k_{1}}{x_{0} y_{0}}, \\
& b_{1}=\frac{k_{2}\left(w_{0}+x_{0}\right)}{2\left(2 d_{1} x_{0}+2 k_{1}+k_{2}\right)}, \quad c_{1}=\frac{2\left(2 c_{2} c_{3} k_{1}\left(4 d_{1} x_{0}+4 k_{1}+k_{2}\right)+k_{8}\right)}{c_{3} k_{2}\left(4 d_{1} x_{0}+4 k_{1}+3 k_{2}\right)}, d_{2}=\frac{c_{2} k_{1}}{y_{0}}, \\
& R=2\left(w_{0}+x_{0}\right) \text { and } \rho=\frac{2 d_{1} x_{0}+2 k_{1}+k_{2}}{w_{0}},
\end{aligned}
$$

where $\mathrm{k}_{1}, \mathrm{k}_{2}, \mathrm{k}_{8}, w_{0}, \mathrm{y}_{0}$ and $\mathrm{x}_{0}$ are positive real numbers. The equilibrium points are given by

$$
\begin{aligned}
& P_{0}=\left(w_{0}, x_{0}, y_{0}, \frac{2 c_{2} c_{3} k_{1}\left(4 d_{1} x_{0}+4 k_{1}+k_{2}\right)+k_{8}}{d_{3}\left(4 d_{1} x_{0}+4 k_{1}+3 k_{2}\right)}\right), \\
& P_{1}=\left(\frac{k_{2} w_{0}}{4 d_{1} x_{0}+4 k_{1}+2 k_{2}}, \frac{k_{2} x_{0}}{4 d_{1} x_{0}+4 k_{1}+2 k_{2}}, y_{0}, \frac{k_{8}}{8 d_{1} d_{3} x_{0}+8 d_{3} k_{1}+4 d_{3} k_{2}}\right) .
\end{aligned}
$$

Proof. In any case, since all the parameters are positive and the points of interest are in $\Gamma$, the equilibrium points for the differential system (1.1) must satisfy the system

$$
\begin{array}{r}
a_{1} R y+\left(b_{1}+w+x\right)(R(v-\rho)+\rho(w+x))=0 \\
v w-x\left(a_{3} y+d_{1}\right)=0 \\
\left(b_{2}+y\right)\left(\left(b_{1}+w+x\right)\left(d_{2}-a_{3} c_{2} x\right)-a_{1} c_{1} w\right)+a_{2} z\left(b_{1}+w+x\right)=0 \\
-a_{2} c_{3} y+b_{2} d_{3}+d_{3} y=0 .
\end{array}
$$

Assume that $P_{0}=\left(w_{0}, x_{0}, y_{0}, z_{0}\right) \in \Gamma$ is an equilibrium point satisfying system (3.1). From fourth equation we have that $y_{0}=\frac{b_{2} d_{3}}{k_{1}}$ where $k_{1}=a_{2} c_{3}-d_{3}>0$, that is, $a_{2}=\frac{k_{1}+d_{3}}{c_{2}}$. Substituting $y_{0}$ in the other equations, the system (3.1) at $\mathrm{P}_{0}$ simplifies to

$$
\begin{array}{r}
a_{1} b_{2} d_{3} R+k_{1}\left(b_{1}+w_{0}+x_{0}\right)\left(R(v-\rho)+\rho\left(w_{0}+x_{0}\right)\right)=0, \\
v k_{1} w_{0}-x_{0}\left(a_{3} b_{2} d_{3}+d_{1} k_{1}\right)=0, \\
c_{3} b_{2}\left(\left(b_{1}+w_{0}+x_{0}\right)\left(d_{2}-a_{3} c_{2} x_{0}\right)-a_{1} c_{1} w_{0}\right)+k_{1} z_{0}\left(b_{1}+w_{0}+x_{0}\right)=0 .
\end{array}
$$

Solving second equation for $w_{0}$, we have $w_{0}=\frac{x_{0}\left(a_{3} b_{2} d_{3}+d_{1} k_{1}\right)}{k_{1} v}$. Substituting $w_{0}$ in the other equations, the system (3.1) at $\mathrm{P}_{0}$ becomes

$$
\begin{array}{r}
v^{2} a_{1} b_{2} d_{3} k_{1} R+\left(a_{3} b_{2} d_{3} x_{0}+k_{1} v\left(b_{1}+x_{0}\right)+d_{1} k_{1} x_{0}\right)\left(a_{3} b_{2} d_{3} \rho x_{0}+k_{1}\left(\rho x_{0}\left(d_{1}+v\right)+v R(v-\rho)\right)\right)=0, \\
\left(\left(a_{3} b_{2} d_{3} x_{0}+k_{1} v\left(b_{1}+x_{0}\right)+d_{1} k_{1} x_{0}\right)\left(b_{2} c_{3}\left(d_{2}-a_{3} c_{2} x_{0}\right)+k_{1} z_{0}\right)-a_{1} b_{2} c_{1} c_{3} x_{0}\left(a_{3} b_{2} d_{3}+d_{1} k_{1}\right)\right)=0 .
\end{array}
$$

Solving second equation in (3.2) for $z_{0}$ we have

$$
z_{0}=\frac{b_{2} c_{3}}{k_{1}}\left(\frac{a_{1} c_{1} x_{0}\left(a_{3} b_{2} d_{3}+d_{1} k_{1}\right)}{a_{3} b_{2} d_{3} x_{0}+k_{1} v\left(b_{1}+x_{0}\right)+d_{1} k_{1} x_{0}}+a_{3} c_{2} x_{0}-d_{2}\right) .
$$


Substituting $z_{0}$ in first equation in (3.2) we have that the system (3.1) simplifies to

$$
R\left(\frac{a_{1} b_{2} d_{3}}{k_{1}}+b_{1}(v-\rho)\right)+\frac{x_{0}\left(b_{1} \rho+R(v-\rho)\right)\left(a_{3} b_{2} d_{3}+k_{1}\left(d_{1}+v\right)\right)}{k_{1} v}+\frac{\rho x_{0}^{2}\left(a_{3} b_{2} d_{3}+k_{1}\left(d_{1}+v\right)\right)^{2}}{k_{1}^{2} v^{2}}=0 .
$$

Now, assume that $R(v-\rho)+b_{1} \rho<0$. Then there is a positive real number $k_{2}$ such that $R(v-\rho)+b_{1} \rho=$ $-k_{2}$. Set $\rho=k_{3}+v$ for some $k_{3}>0$ and $k_{3}=\frac{a_{1} b_{2} d_{3}}{b_{1} k_{1}}$. Then, $R=\frac{b_{1}\left(a_{1} b_{2} d_{3}+k_{1}\left(b_{1} v+k_{2}\right)\right)}{a_{1} b_{2} d_{3}}$. Substituting $R$ and $\rho$ in the above quadratic equation with respect to $x_{0}$ it has a positive root $x_{0}$ given by

$$
x_{0}=\frac{b_{1} k_{1}^{2} k_{2} v}{\left(a_{1} b_{2} d_{3}+b_{1} k_{1} v\right)\left(a_{3} b_{2} d_{3}+k_{1}\left(d_{1}+v\right)\right)} .
$$

Finally, from (3.3) we have that $z_{0}>0$ if we take $d_{2}=\frac{a_{1} c_{1} x_{0}\left(a_{3} b_{2} d_{3}+d_{1} k_{1}\right)}{a_{3} b_{2} d_{3} x_{0}+k_{1} v\left(b_{1}+x_{0}\right)+d_{1} k_{1} x_{0}}$. Hence we have proved the claim (i).

We now prove claim (ii). We suppose that $P_{0}=\left(w_{0}, x_{0}, y_{0}, z_{0}\right) \in \Gamma$ satisfies (3.1). Solving first, second, third, and fourth equation in the variables $a_{1}, a_{2}, a_{3}$, and $c_{1}$, respectively, one gets

$$
\begin{aligned}
& a_{1}=-\frac{\left(b_{1}+w_{0}+x_{0}\right)\left(R(v-\rho)+\rho\left(w_{0}+x_{0}\right)\right)}{R y_{0}}, a_{2}=\frac{d_{3}\left(b_{2}+y_{0}\right)}{c_{3} y_{0}}, a_{3}=\frac{v w_{0}-d_{1} x_{0}}{x_{0} y_{0}}, \text { and } \\
& c_{1}=-\frac{R\left(c_{2} c_{3}\left(d_{1} x_{0}-v w_{0}\right)+c_{3} d_{2} y_{0}+d_{3} z_{0}\right)}{c_{3} w_{0}\left(v R+\rho\left(-R+w_{0}+x_{0}\right)\right)} .
\end{aligned}
$$

Taking $v=\frac{d_{1} x_{0}+k_{1}}{w_{0}}, d_{2}=\frac{c_{2} k_{1}}{y_{0}}, R=2\left(w_{0}+x_{0}\right)$, and $\rho=\frac{2 d_{1} x_{0}+2 k_{1}+k_{2}}{w_{0}}$, where $k_{1}, k_{2}>0$ we have that

$$
a_{1}=\frac{k_{2}\left(b_{1}+w_{0}+x_{0}\right)}{2 w_{0} y_{0}}, \quad a_{2}=\frac{d_{3}\left(b_{2}+y_{0}\right)}{c_{3} y_{0}}, \quad a_{3}=\frac{k_{1}}{x_{0} y_{0}}, \quad c_{1}=\frac{2 d_{3} z_{0}}{c_{3} k_{2}}
$$

and $\mathrm{P}_{0} \in \Gamma$ is an equilibrium point for the differential system (1.1). Hence under these conditions for the parameters $a_{1}, a_{2}, a_{3}$, and $c_{1}$ the system (3.1) takes the form

$$
\begin{array}{r}
y_{0}\left(b_{1}+w+x\right)\left(2 d_{1} x_{0}\left(w-w_{0}+x-x_{0}\right)+2 k_{1}\left(w-w_{0}+x-x_{0}\right)+k_{2}\left(w-2 w_{0}+x-2 x_{0}\right)\right) \\
+k_{2} y\left(w_{0}+x_{0}\right)\left(b_{1}+w_{0}+x_{0}\right)=0 \\
x_{0} y_{0} w\left(d_{1} x_{0}+k_{1}\right)-w_{0} x\left(x_{0} y_{0} d_{1}+k_{1} y\right)=0 \\
\frac{\left(b_{1}+w+x\right)\left(d_{3} x_{0} z\left(b_{2}+y_{0}\right)-c_{2} c_{3} k_{1}\left(b_{2}+y\right)\left(x-x_{0}\right)\right)}{x_{0}}-\frac{d_{3} w z_{0}\left(b_{2}+y\right)\left(b_{1}+w_{0}+x_{0}\right)}{w_{0}}=0 \\
b_{2} d_{3}\left(y_{0}-y\right)=0
\end{array}
$$

Assume that $\mathrm{P}_{1}=\left(w_{1}, \mathrm{x}_{1}, \mathrm{y}_{1}, z_{1}\right) \in \Gamma \backslash\left\{\mathrm{P}_{0}\right\}$ satisfies (3.4). We will give conditions to find expressions for $P_{1}$. Since all the parameters are positive, from fourth equation we have $y_{1}=y_{0}$ and substituting $y_{1}$ in the other equations we have that system (3.4) simplifies to

$$
\begin{aligned}
&\left(w_{1}-w_{0}+x_{1}-x_{0}\right)\left(2 k_{1}\left(w_{1}+x_{1}\right)+k_{2}\left(w_{1}-w_{0}+x_{1}\right)\right. \\
&\left.+\left(-k_{2}+2 d_{1}\left(w_{1}+x_{1}\right)\right) x_{0}+b_{1}\left(2 k_{1}+k_{2}+2 d_{1} x_{0}\right)\right)=0 \\
& w_{1} x_{0}-w_{1} x_{1}=0 \\
& \\
&-w_{0}\left(b_{1}+w_{1}+x_{1}\right)\left(c_{2} c_{3} k_{1}\left(x_{1}-x_{0}\right)-d_{3} x_{0} z_{1}\right)-d_{3} w_{1} x_{0} z_{0}\left(b_{1}+w_{0}+x_{0}\right)=0 .
\end{aligned}
$$

Therefore, from second equation $w_{1}=\frac{w_{0} x_{1}}{x_{0}}$. Substituting $w_{1}$ in first equation and solving the resulting equation for $x_{1}$, we have $x_{1}=\frac{x_{0}\left(k_{2}\left(w_{0}+x_{0}\right)-b_{1}\left(2 d_{1} x_{0}+2 k_{1}+k_{2}\right)\right)}{\left(w_{0}+x_{0}\right)\left(2 d_{1} x_{0}+2 k_{1}+k_{2}\right)}$. Since $x_{1}$ must be positive, we have $k_{2}\left(w_{0}+\right.$ $\left.x_{0}\right)-b_{1}\left(2 d_{1} x_{0}+2 k_{1}+k_{2}\right)=k_{9}$ for some $k_{9}>0$. Take $k_{9}=\frac{1}{2} k_{2}\left(w_{0}+x_{0}\right)$. Then, substituting $w_{1}$ and $x_{1}$ in third equation we have $z_{1}=\frac{d_{3} z_{0}\left(4 d_{1} x_{0}+4 k_{1}+3 k_{2}\right)-2 c_{2} c_{3} k_{1}\left(4 d_{1} x_{0}+4 k_{1}+k_{2}\right)}{4 d_{3}\left(2 d_{1} x_{0}+2 k_{1}+k_{2}\right)}$. Since $z_{1}$ must be positive, we take $k_{8}>0$ such that $-2 c_{2} c_{3} k_{1}\left(4 k_{1}+k_{2}+4 d_{1} x_{0}\right)+d_{3}\left(4 k_{1}+3 k_{2}+4 d_{1} x_{0}\right) z_{0}=k_{8}$. These conditions guarantee that $P_{1}$ and $P_{0}$ are as claim (ii) states. 


\subsection{Dynamics of one equilibrium point}

Lemma 3.2. If the hypothesis of Lemma 3.1 (i) are satisfied and

$$
\begin{aligned}
& k_{1}=d_{3}, \quad a_{1}=\frac{b_{1} v}{b_{2}}, \quad a_{2}=\frac{2 d_{3}}{c_{3}}, \quad a_{3}=\frac{3 v}{b_{2}}, \quad d_{1}=2 v, \quad k_{2}=35 b_{1} v, \quad c_{1}=\frac{2738 c_{2}}{395}, \\
& d_{2}=\frac{1295 b_{1} c_{2} v}{237 b_{2}}, \quad b_{20}:=b_{1} c_{2}\left(\frac{27983097891 d_{3}}{16779436846 v}+\frac{28749}{37756}\right), \text { and } v \neq \frac{30421101094081 d_{3}}{16443848109080},
\end{aligned}
$$

then the eigenvalues of the linear approximation $\mathrm{M}_{0}\left(\mathrm{~b}_{20}\right)$ of system (1.1) at $\mathrm{P}_{0}$ are

$$
\begin{aligned}
& \lambda_{1,2}= \pm i \frac{\sqrt{\frac{41948592115}{237}} v}{8214}, \\
& \lambda_{3,4}=-\frac{10961510700 d_{3} v}{7038482959 d_{3} \pm \sqrt{745681} \sqrt{d_{3}\left(30421101094081 d_{3}-16443848109080 v\right)}} .
\end{aligned}
$$

Proof. Assume that the hypothesis in Lemma 3.1 (i) are valid. To simplify the analysis we set $k_{1}=d_{3}, a_{1}=$ $\frac{b_{1} v}{b_{2}}$ and $a_{3}=\frac{d_{1}+v}{b_{2}}$. Then the characteristic polynomial for the linear approximation $M\left(P_{0}\right)$ of system (1.1) at $P_{0}$ is $\operatorname{pol}_{0}\left(\lambda, b_{2}\right)=\lambda^{4}+A_{1} \lambda^{3}+A_{2} \lambda^{2}+A_{3} \lambda+A_{4}$, where

$$
\begin{aligned}
& A_{1}=\frac{4 b_{2}\left(2 d_{1}+v\right) E_{0}-c_{2} k_{2}\left(d_{1}+v\right)\left(2 b_{1} v+k_{2}\right)^{2}}{8 b_{2}\left(d_{1}+v\right)\left(2 b_{1} v+k_{2}\right)^{2}}, \\
& A_{2}=\frac{k_{2}\left(4 v\left(2 d_{1}+v\right) E_{1}+c_{2}\left(d_{1}+v\right)\left(2 b_{1} v+k_{2}\right) E_{2}\right)}{16 b_{2}\left(d_{1}+v\right)^{2}\left(2 b_{1} v+k_{2}\right)^{3}}, \\
& A_{3}=\frac{k_{2}\left(2 d_{1}+v\right)\left(4 b_{1} c_{1} v^{3}\left(8 b_{1}^{2} v\left(2 d_{1}+v\right)-k_{2}^{2}\right)+c_{2}\left(2 b_{1} v+k_{2}\right) E_{3}\right)}{16 b_{2}\left(d_{1}+v\right)\left(2 b_{1} v+k_{2}\right)^{3}}, \\
& A_{4}=\frac{c_{2} d_{3} k_{2}^{3} v\left(2 d_{1}+v\right)}{8 b_{2}\left(2 b_{1} v+k_{2}\right)^{2}} \\
& \mathbf{E}_{0}=v\left(8 b_{1}^{2} v^{2}+8 b_{1} k_{2} v+3 k_{2}^{2}\right)+2 d_{1}\left(2 b_{1} v+k_{2}\right)^{2}, \\
& E_{1}=b_{1} c_{1} v\left(8 b_{1}^{2} v^{3}-d_{1}\left(k_{2}-2 b_{1} v\right)\left(4 b_{1} v+k_{2}\right)-k_{2}^{2} v\right)+4 b_{2} k_{2}\left(d_{1}+v\right)^{2}\left(2 b_{1} v+k_{2}\right), \\
& E_{2}=v\left(v\left(8 b_{1}^{2} v^{2}+8 b_{1} k_{2} v+k_{2}^{2}\right)+2 d_{3}\left(2 b_{1} v+k_{2}\right)^{2}\right)+2 d_{1}\left(d_{3}\left(2 b_{1} v+k_{2}\right)^{2}+4 b_{1} v^{2}\left(b_{1} v+k_{2}\right)\right), \\
& \mathbf{E}_{3}=v\left(16 b_{1}^{2} v^{3}+8 b_{1} v^{2}\left(b_{1} d_{3}+k_{2}\right)+8 b_{1} d_{3} k_{2} v+3 d_{3} k_{2}^{2}\right)+2 d_{1}\left(2 b_{1} v+k_{2}\right)\left(2 b_{1} d_{3} v+4 b_{1} v^{2}+d_{3} k_{2}\right) .
\end{aligned}
$$

Therefore, setting $d_{1}=2 v$ expression (2.1) becomes the function, in the variable $b_{2}$,

$$
\mathbf{E Q}\left(b_{2}\right)=\frac{5 k_{2}^{2}\left(360 b_{2}\left(2 b_{1} v+k_{2}\right)^{2}\left(G_{0}\right)^{2}-\frac{\left(G_{1}\right)\left(G_{2}\right)\left(G_{3}\right)}{v}+36 c_{2} d_{3} k_{2} v^{2}\left(2 b_{1} v+k_{2}\right)^{2}\left(G_{4}\right)^{2}\right)}{165888 b_{2}^{3}\left(2 b_{1} v+k_{2}\right)^{8}}
$$

where

$$
\begin{aligned}
\mathbf{G}_{0}= & 4 b_{1} c_{1} v^{3}\left(40 b_{1}^{2} v^{2}-k_{2}^{2}\right)+c_{2} v\left(2 b_{1} v+k_{2}\right)\left(48 b_{1}^{2} v^{3}+24 b_{1} v^{2}\left(b_{1} d_{3}+k_{2}\right)+24 b_{1} d_{3} k_{2} v+7 d_{3} k_{2}^{2}\right), \\
G_{1}= & 20 b_{2} v\left(24 b_{1}^{2} v^{2}+24 b_{1} k_{2} v+7 k_{2}^{2}\right)-3 c_{2} k_{2}\left(2 b_{1} v+k_{2}\right)^{2}, \\
G_{2}= & 4 b_{1} c_{1} v^{3}\left(40 b_{1}^{2} v^{2}-k_{2}^{2}\right)+c_{2} v\left(2 b_{1} v+k_{2}\right)\left(48 b_{1}^{2} v^{3}+24 b_{1} v^{2}\left(b_{1} d_{3}+k_{2}\right)+24 b_{1} d_{3} k_{2} v+7 d_{3} k_{2}^{2}\right), \\
G_{3}= & 20 v^{3}\left(b_{1} c_{1}\left(24 b_{1}^{2} v^{2}-4 b_{1} k_{2} v-3 k_{2}^{2}\right)+36 b_{2} k_{2}\left(2 b_{1} v+k_{2}\right)\right) \\
& +3 c_{2} v\left(2 b_{1} v+k_{2}\right)\left(v\left(24 b_{1}^{2} v^{2}+24 b_{1} k_{2} v+k_{2}^{2}\right)+6 d_{3}\left(2 b_{1} v+k_{2}\right)^{2}\right), \\
G_{4}= & 3 c_{2} k_{2}\left(2 b_{1} v+k_{2}\right)^{2}-20 b_{2} v\left(24 b_{1}^{2} v^{2}+24 b_{1} k_{2} v+7 k_{2}^{2}\right) .
\end{aligned}
$$

Now, if $k_{2}=35 b_{1} v$, then 


$$
\mathbf{E Q}\left(b_{2}\right)=\frac{30625 b_{1} v^{4}\left(12 v^{2}\left(2738 c_{2}-395 c_{1}\right) G_{5}+9154363015180278 b_{1}^{2} c_{2}^{3} d_{3}^{2}+G_{6}\right)}{582678191652046848 b_{2}^{3}}
$$

where

$$
\begin{aligned}
& \mathrm{G}_{5}=-28749 \mathrm{~b}_{1}^{2} c_{2}\left(75820 c_{1}-231879 c_{2}\right)+4 b_{1} b_{2}\left(598861900 c_{1}+5322323571 c_{2}\right)-35203694400 b_{2}^{2} \\
& G_{6}=37 b_{1} c_{2} d_{3} v\left(37756 b_{2}\left(598861900 c_{1}-8080460229 c_{2}\right)-28749 b_{1} c_{2}\left(832468060 c_{1}-9699735333 c_{2}\right)\right) .
\end{aligned}
$$

Taking $c_{1}=\frac{2738 c_{2}}{395}$, we have

$$
\begin{aligned}
A_{1} & =\frac{105577244385 d_{3} v}{18375071202 d_{3}+8389718423 v}, \\
A_{2} & =\frac{5 v^{2}\left(417079125059936669046 d_{3}+70387375217225606929 v\right)}{15990341652\left(18375071202 d_{3}+8389718423 v\right)}, \\
A_{3} & =\frac{18686990554143624575 d_{3} v^{3}}{67469796\left(18375071202 d_{3}+8389718423 v\right)}, \\
A_{4} & =\frac{214375 d_{3} v^{3}}{10952\left(\frac{27983097891 d_{3}}{16779436846 v}+\frac{28749}{37756}\right)} .
\end{aligned}
$$

Hence, (2.1) simplifies to

$$
\operatorname{EQ}\left(b_{2}\right)=\frac{30625 b_{1}^{2} c_{2}^{2} d_{3} v^{4}\left(8389718423 v\left(28749 b_{1} c_{2}-37756 b_{2}\right)+528264921986298 b_{1} c_{2} d_{3}\right)}{33624234580359168 b_{2}^{3}}
$$

and $\mathbf{E Q}\left(b_{20}\right)=0$, where $b_{20}=b_{1} c_{2}\left(\frac{27983097891 d_{3}}{16779436846 v}+\frac{28749}{37756}\right)$. The proof follows from Lemma 2.1 (ii).

Lemma 3.3. If the hypothesis of Lemma 3.2 are valid and $\mathrm{d}_{3}=10, \mathrm{c}_{2}=\frac{1}{10}, \mathrm{c}_{3}=\frac{1}{2}, \mathrm{~b}_{1}=1$, then there exist $v_{0}, v_{1}$ positive real numbers such that the first Lyapunov coefficient for the system (1.1) at $\mathrm{P}_{0}$ is positive if either $0<v<v_{0}$ or $v>v_{1}$, and it is negative if $v_{0}<v<v_{1}$. Moreover, the transversality condition holds: $\frac{\mathrm{d} \operatorname{Re}\left(\lambda_{1,2}\right)}{\mathrm{d} \mathrm{b}_{2}}\left(\mathrm{~b}_{20}\right) \neq 0$.

Proof. If the hypothesis are valid, using Proposition 2.2 for the transversality condition and the Kuznetsov formulae (see $[2,7,8]$ ) for the first Lyapunov coefficient, the Mathematica software allows to get by a direct calculation:

$$
\frac{\mathrm{d} \operatorname{Re}\left(\lambda_{1,2}\right)}{\mathrm{db}_{2}}\left(\mathrm{~b}_{20}\right)=-\frac{22070466729033979537997184429118254339826180728050 v^{3}}{\mathrm{~s}_{1}(v)} \neq 0,
$$

where

$$
\begin{aligned}
s_{1}(v)= & 118072143\left(4954382589970505556215207055672412811041 v^{2}\right. \\
& -153100675464069678846765525147766678141320 v \\
& +31089982892973169931537646413835378802713600) .
\end{aligned}
$$

And that $\ell_{1}\left(P_{0}, b_{20}\right)=-\frac{N_{0}}{N_{1}}$, where

$$
\begin{aligned}
\mathrm{N}_{0}= & 351936876086128034645 \sqrt{\frac{3313938777085}{3}} v \\
& \times\left(66100655705229180407012562120741326878526172401290087447529295055585272751069329615626823335 v^{5}\right. \\
& -5976171976577332106606135926837595167406791205883486650756176985676594548604919209508115326404 v^{4} \\
& -76596224628557684030051225997470099342353475309263857165708452193625218362629656244443950086020 v^{3}
\end{aligned}
$$




$$
\begin{aligned}
& -6170102643931266987022838519078935939108816686869336913207841796659032566131751047615550510714400 v^{2} \\
& -5653648766502639134187836199614462873235179102144729125824880862755619868039896864986829728800000 v \\
& +1574303131909464229944026194947529078952694682667394044189255748718719649104366999659556460800000) ; \\
N_{1}= & 6573938\left(876241431697782963899717150349773 v^{2}+75662348547641698853038918874340 v\right. \\
& +828574316667181373417609314915800)\left(4954382589970505556215207055672412811041 v^{2}\right. \\
& -153100675464069678846765525147766678141320 v+31089982892973169931537646413835378802713600) \\
& \times\left(4954382589970505556215207055672412811041 v^{2}+124490364366450894812917026926295506809680 v\right. \\
& +8258827593974963520933437147080834871447100) .
\end{aligned}
$$

A numeric calculation shows that the positive roots of $\ell_{1}\left(P_{0}, b_{20}\right)$ are $v_{0} \approx 0.223694$ and $v_{1} \approx 108.971$ which satisfy the desired properties (see Fig. 1).
a) $\ell_{1}\left(P_{0}\right)<0$

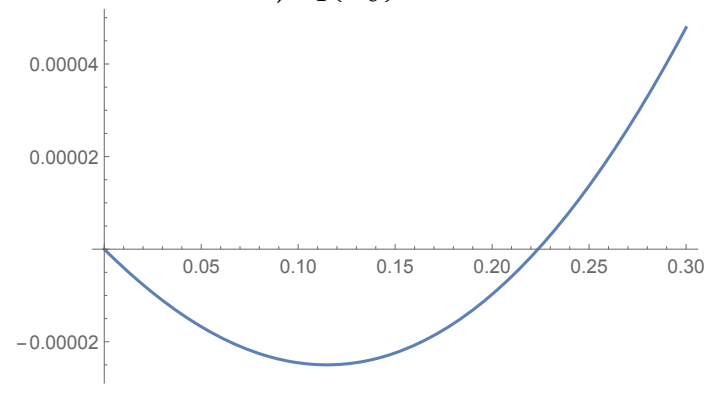

b) $\ell_{1}\left(P_{0}\right)>0$ or $\ell_{1}\left(P_{0}\right)<0$

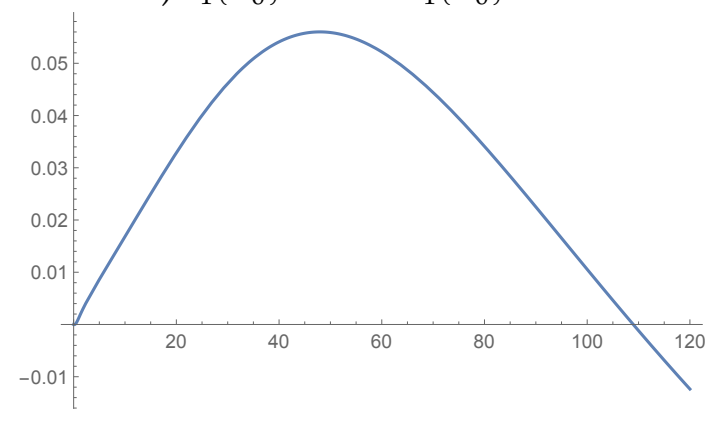

Figure 1: First Lyapunov coefficient.

Theorem 3.4 ( $\mathrm{f}_{1}$ Holling II, one equilibrium point). Assume that the parameters of system (1.1) satisfy the hypothesis of Lemma 3.3. Then, the system exhibits a Hopf bifurcation at $\mathrm{P}_{0}$ with respect to the parameter $\mathrm{b}_{2}$ and its bifurcation value is $b_{20}$. This bifurcation is supercritical if $0<v<v_{0}$ or $v>v_{1}$, and it is subcritical if $v_{0}<v<v_{1}$.

Proof. It follows from Lemma 3.2 and the Andronov-Hopf Theorem [8, 12, 14].

\subsection{Dynamics of two equilibria}

Lemma 3.5. If the hypothesis of Lemma 3.1 (ii) are satisfied and

$$
\begin{aligned}
& c_{2}=\frac{365 y_{0}}{44 w_{0}}, d_{1}=\frac{2920 d_{3}}{87}, k_{1}=\frac{365 d_{3} w_{0}}{87}, k_{2}=\frac{2920 d_{3} w_{0}}{87}, \\
& k_{8}=\frac{97254250 b_{2} c_{3} d_{3}^{2} w_{0}}{7569}, \text { and } b_{20}:=\frac{2361971095625 y_{0}}{131632971184},
\end{aligned}
$$

then the eigenvalues of the linear approximation $\mathrm{M}_{0}\left(\mathrm{~b}_{20}\right)$ of system (1.1) at $\mathrm{P}_{0}$ are

$$
\lambda_{1,2}= \pm i 365 \sqrt{\frac{2525226}{1668424897}} d_{3}, \quad \lambda_{3,4}=-\frac{73(1056 \pm i \sqrt{677949239}) d_{3}}{22968} .
$$


Proof. The proof is similar to the given for Lemma 3.1 (i). Assume that the hypothesis in Lemma 3.1 (ii) hold. In order to simplify the calculations set $x_{0}=w_{0}$,

$$
\mathrm{k}_{8}=\frac{\mathrm{b}_{2} \mathrm{c}_{3}\left(24 \mathrm{~d}_{1}^{2} w_{0}^{2}+18 \mathrm{~d}_{1} \mathrm{k}_{2} w_{0}+24 \mathrm{k}_{1}^{2}+18 \mathrm{k}_{1} \mathrm{k}_{2}+\mathrm{k}_{2}^{2}\right)}{4 w_{0}} \text { and } \mathrm{c}_{2}=\frac{y_{0}\left(24 \mathrm{~d}_{1}^{2} w_{0}^{2}+18 \mathrm{~d}_{1} \mathrm{k}_{2} w_{0}+24 \mathrm{k}_{1}^{2}+18 \mathrm{k}_{1} \mathrm{k}_{2}+\mathrm{k}_{2}^{2}\right)}{8 \mathrm{k}_{1} w_{0}\left(4 \mathrm{~d}_{1} w_{0}+4 \mathrm{k}_{1}+\mathrm{k}_{2}\right)} .
$$

Now making $k_{1}=\frac{k_{2}}{8}$ and $k_{2}=d_{1} w_{0}$ we have that the linear approximation at $P_{0}$ for the system (1.1) has characteristic polynomial

$$
\operatorname{pol}_{0}\left(\lambda, b_{2}\right)=\lambda^{4}+A_{10} \lambda^{3}+A_{20} \lambda^{2}+A_{30} \lambda+A_{40}
$$

where

$$
\begin{aligned}
& A_{10}=\frac{d_{1}}{5}, \quad A_{20}=\frac{d_{1}\left(803 b_{2}\left(11 d_{1}+48 d_{3}\right)-20233 d_{1} y_{0}\right)}{25344 y_{0}}, \\
& A_{30}=\frac{73 d_{1}^{2}\left(22 b_{2}\left(d_{1}+3304 d_{3}\right)-38261 d_{1} y_{0}\right)}{2027520 y_{0}}, \text { and } A_{40}=\frac{10439 b_{2} d_{1}^{3} d_{3}}{5120 y_{0}} .
\end{aligned}
$$

In this case, expression (2.1) is $\mathbf{E Q}=\frac{73 \mathrm{~d}_{1}^{4} \mathrm{~T}_{1}}{20554186752000 y_{0}^{2}}$, where

$$
\begin{aligned}
T_{1}= & -176660 b_{2}^{2}\left(d_{1}+3304 d_{3}\right)\left(87 d_{1}-2920 d_{3}\right) \\
& +572 b_{2} d_{1} y_{0}\left(46255055 d_{1}-3097232904 d_{3}\right)+472394219125 d_{1}^{2} y_{0}^{2} .
\end{aligned}
$$

Taking $d_{1}=\frac{2920 d_{3}}{87}$, we have that all the coefficients of $\operatorname{pol}_{0}\left(\lambda, b_{2}\right)$ are positive and $\mathbf{E Q}\left(b_{20}\right)=0$, where

$$
\mathrm{b}_{20}=\frac{2361971095625 \mathrm{y}_{0}}{131632971184} \text {. }
$$

The proof concludes using Lemma 2.1 (ii).

Lemma 3.6. Under the hypothesis of Lemma 3.5 the first Lyapunov coefficient for the system (1.1) at $\mathrm{P}_{0}$ is negative. Moreover, the transversality condition holds: $\frac{\mathrm{d} \operatorname{Re}\left(\lambda_{1,2}\right)}{\mathrm{db}_{2}}\left(\mathrm{~b}_{20}\right) \neq 0$.

Proof. Similarly to the proof of Lemma 3.3, a calculation proves that

$$
\frac{\mathrm{d} \operatorname{Re}\left(\lambda_{1,2}\right)}{\mathrm{db} \mathrm{b}_{2}}\left(\mathrm{~b}_{20}\right)=-\frac{9287262564940698723330882878656 \mathrm{~d}_{3}}{41362560595546268863797036236319 \mathrm{y}_{0}} \neq 0
$$

and that the first Lyapunov coefficient is $\ell_{1}\left(P_{0}\right)=-\frac{N_{0}}{N_{1}}$, where

$$
\begin{aligned}
\mathrm{N}_{0}= & 8507365617004380447171374616484388706047917265654519261322268539426856405245286959597 \\
& 329625166819212900509434773841920 \sqrt{\frac{110116043202}{38261}} ; \\
\mathrm{N}_{1}= & 9430175494429761358556394005879301699402603777598726258708502056302804492108705495457 \\
& \times\left(48807341380019929\left(777031385380692461328125 c_{3}^{2}+186770227571152422912\right) y_{0}^{2}\right. \\
& \left.+3353725607660164343881855230232467456 w_{0}^{2}\right) .
\end{aligned}
$$

Under the hypothesis of Lemma 3.1 (ii), a direct calculation shows that the characteristic polynomial for the linear approximation of system (1.1) at $P_{1}$ has constant term

$$
A_{41}=-\frac{b_{2} d_{3} k_{8}\left(d_{1} x_{0}+k_{1}\right)\left(4 d_{1} x_{0}+4 k_{1}+k_{2}\right)}{32 c_{3} w_{0} x_{0} y_{0}\left(b_{2}+y_{0}\right)\left(2 d_{1} x_{0}+2 k_{1}+k_{2}\right)},
$$

which is negative since all the parameters are positive. Hence from the Routh-Hurwitz test we have that the equilibrium point $P_{1}$ is locally unstable. In summary, we have proved next result, which follows from Lemma 3.6 and the Andronov-Hopf Theorem.

Theorem 3.7 ( $f_{1}$ Holling II, two equilibrium points). Assume that the parameters of system (1.1) satisfy the hypothesis of Lemma 3.5. Then, $\mathrm{P}_{1}$ is locally unstable and the system (1.1) exhibits a supercritical Hopf bifurcation at $\mathrm{P}_{0}$ with respect to the parameter $\mathrm{b}_{2}$ and its bifurcation value is $\mathrm{b}_{20}$. 


\section{Case $f_{1}(w, x)$ Holling IV}

Lemma 4.1. The differential system (1.1) has:

i) An equilibrium point in $\Gamma$ if

$$
\begin{aligned}
& a_{2}=\frac{d_{3}+k_{1}}{c_{3}}, \quad b_{1}=\frac{k_{1}^{2} v^{2}+k_{4}}{\left(a_{3} b_{2} d_{3}+d_{1} k_{1}\right)^{2}}, \quad d_{2}=\frac{a_{1} c_{1} k_{1} k_{4} v\left(a_{3} b_{2} d_{3}+d_{1} k_{1}\right)}{\left(k_{1}^{2} v^{2}+k_{4}\right)^{2}}, \rho=\frac{a_{1} b_{2} d_{3}\left(a_{3} b_{2} d_{3}+d_{1} k_{1}\right)^{2}}{k_{1}\left(k_{1}^{2} v^{2}+k_{4}\right)}+v \text { and } \\
& R=\frac{\left(k_{1}^{2} v^{2}+k_{4}\right)\left(a_{3} b_{2} d_{3}+k_{1}\left(d_{1}+v\right)\right)\left(a_{1} b_{2} d_{3}\left(a_{3} b_{2} d_{3}+d_{1} k_{1}\right)^{2}+k_{1}^{3} v^{3}+k_{1} k_{4} v\right)}{a_{1} b_{2} d_{3} k_{1} v\left(a_{3} b_{2} d_{3}+d_{1} k_{1}\right)^{4}},
\end{aligned}
$$

where $k_{1}, k_{4}$ are positive real numbers. The equilibrium is given by

$$
P_{0}=\left(\frac{k_{4}}{a_{3} b_{2} d_{3} k_{1} v+d_{1} k_{1}^{2} v}, \frac{k_{4}}{\left(a_{3} b_{2} d_{3}+d_{1} k_{1}\right)^{2}}, \frac{b_{2} d_{3}}{k_{1}}, \frac{a_{3} b_{2} c_{2} c_{3} k_{4}}{k_{1}\left(a_{3} b_{2} d_{3}+d_{1} k_{1}\right)^{2}}\right) .
$$

ii) Two equilibrium points in $\Gamma$ if

$$
\begin{aligned}
& \mathrm{a}_{1}=\frac{\left(\mathrm{d}_{1} w_{0}^{4}+2 \mathrm{k}_{1} w_{0}^{2}+2 \mathrm{k}_{3}\right)\left(6 \mathrm{~d}_{1} w_{0}^{4}+12 \mathrm{k}_{1} w_{0}^{2}+7 \mathrm{k}_{3}\right)^{2}}{32 w_{0} \mathrm{y}_{0}\left(\mathrm{~d}_{1} w_{0}^{4}+2 \mathrm{k}_{1} w_{0}^{2}+\mathrm{k}_{3}\right)^{2}}, \mathrm{a}_{2}=\frac{\mathrm{d}_{3}\left(\mathrm{~b}_{2}+\mathrm{y}_{0}\right)}{\mathrm{c}_{3} \mathrm{y}_{0}}, \mathrm{a}_{3}=\frac{2 \mathrm{k}_{1}}{w_{0}^{2} \mathrm{y}_{0}}, \\
& \mathrm{~b}_{1}=\frac{1}{16} w_{0}^{2}\left(\frac{\mathrm{k}_{3}\left(12 \mathrm{~d}_{1} w_{0}^{4}+24 \mathrm{k}_{1} w_{0}^{2}+13 \mathrm{k}_{3}\right)}{\left(\mathrm{d}_{1} w_{0}^{4}+2 \mathrm{k}_{1} w_{0}^{2}+\mathrm{k}_{3}\right)^{2}}+12\right), \mathrm{d}_{2}=\frac{\mathrm{c}_{2} \mathrm{k}_{1}}{\mathrm{y}_{0}}, \mathrm{R}=w_{0}\left(w_{0}+2\right), \\
& \mathrm{c}_{1}=\frac{w_{0}^{2}\left(2 \mathrm{c}_{2} \mathrm{c}_{3} \mathrm{k}_{1}\left(\mathrm{~d}_{1} w_{0} w^{4}+2 \mathrm{k}_{1} w_{0}^{2}+2 \mathrm{k}_{3}\right)\left(4 \mathrm{~d}_{1} w_{0}^{4}+8 \mathrm{k}_{1} w_{0}^{2}+3 \mathrm{k}_{3}\right)+\mathrm{k}_{5}\right)}{\mathrm{c}_{3} \mathrm{k}_{3}\left(\frac{\mathrm{d}_{1} w_{0}^{4}}{2}+\mathrm{k}_{1} w_{0}^{2}+\mathrm{k}_{3}\right)\left(6 \mathrm{~d}_{1} w_{0}^{4}+12 \mathrm{k}_{1} w_{0}^{2}+7 \mathrm{k}_{3}\right)} \text { and } \rho=\frac{2\left(\mathrm{~d}_{1} w_{0}^{4}+2 \mathrm{k}_{1} w_{0}^{2}+\mathrm{k}_{3}\right)}{w_{0}^{3}},
\end{aligned}
$$

where $w_{0}, y_{0}, k_{1}, k_{3}$ and $k_{5}$ are positive real numbers. The equilibrium points are given by

$$
\begin{aligned}
& P_{0}=\left(w_{0}, \frac{w_{0}^{2}}{2}, y_{0}, \frac{2 c_{2} c_{3} k_{1}\left(d_{1} w_{0}^{4}+2 k_{1} w_{0}^{2}+2 k_{3}\right)\left(4 d_{1} w_{0}^{4}+8 k_{1} w_{0}^{2}+3 k_{3}\right)+k_{5}}{d_{3} k_{3}\left(6 d_{1} w_{0}^{4}+12 k_{1} w_{0}^{2}+7 k_{3}\right)}\right), \\
& P_{1}=\left(\frac{k_{3} w_{0}}{4 \mathrm{~d}_{1} w_{0}^{4}+8 k_{1} w_{0}^{2}+4 k_{3}}, \frac{k_{3} w_{0}^{2}}{8\left(\mathrm{~d}_{1} w_{0}^{4}+2 k_{1} w_{0}^{2}+k_{3}\right)}, y_{0}, \frac{k_{5}}{8 d_{3}\left(d_{1} w_{0}^{4}+2 k_{1} w_{0}^{2}+k_{3}\right)\left(d_{1} w_{0}^{4}+2 k_{1} w_{0}^{2}+2 k_{3}\right)}\right) .
\end{aligned}
$$

iii) Three equilibrium points in $\Gamma$ if

$$
\begin{aligned}
& a_{1}=\frac{3\left(\mathrm{~d}_{1} w_{0}^{4}+2 \mathrm{k}_{1} w_{0}^{2}+2 \mathrm{k}_{3}\right)\left(4 \mathrm{~d}_{1} w_{0}^{4}+8 \mathrm{k}_{1} w_{0}^{2}+5 \mathrm{k}_{3}\right)\left(12 \mathrm{~d}_{1} w_{0}^{4}+24 \mathrm{k}_{1} w_{0}^{2}+13 \mathrm{k}_{3}\right)}{128 w_{0} \mathrm{y}_{0}\left(\mathrm{~d}_{1} w_{0}^{4}+2 \mathrm{k}_{1} w_{0}^{2}+\mathrm{k}_{3}\right)^{2}}, \\
& a_{2}=\frac{d_{3}\left(b_{2}+y_{0}\right)}{c_{3} y_{0}}, \quad a_{3}=\frac{2 k_{1}}{w_{0}^{2} y_{0}}, \quad b_{1}=\frac{3}{64} w_{0}^{2}\left(\frac{k_{3}\left(16 d_{1} w_{0}^{4}+32 k_{1} w_{0}^{2}+17 k_{3}\right)}{\left(d_{1} w_{0}^{4}+2 k_{1} w_{0}^{2}+k_{3}\right)^{2}}+16\right) \text {, } \\
& c_{1}=\frac{w_{0}^{2}\left(4 c_{2} c_{3} k_{1}\left(d_{1} w_{0}^{4}+2 k_{1} w_{0}^{2}+2 k_{3}\right)\left(8 d_{1} w_{0}^{4}+16 k_{1} w_{0}^{2}+7 k_{3}\right)+k_{6}\right)}{3 c_{3} k_{3}\left(\frac{d_{1} w_{0}^{4}}{2}+k_{1} w_{0}^{2}+k_{3}\right)\left(4 d_{1} w_{0}^{4}+8 k_{1} w_{0}^{2}+5 k_{3}\right)}, c_{2}=\frac{c_{2} k_{1}}{y_{0}}, \\
& \mathrm{R}=w_{0}\left(w_{0}+2\right) \text {, and } \rho=\frac{2\left(\mathrm{~d}_{1} w_{0}^{4}+2 \mathrm{k}_{1} w_{0}^{2}+\mathrm{k}_{3}\right)}{w_{0}^{3}} \text {, }
\end{aligned}
$$

where $w_{0}, y_{0}, k_{1}, k_{3}$, and $k_{5}$ are positive real numbers. The equilibrium points are

$$
\begin{aligned}
& \mathrm{P}_{0}=\left(w_{0}, \frac{w_{0}^{2}}{2}, \mathrm{y}_{0}, \frac{4 \mathrm{c}_{2} \mathrm{c}_{3} \mathrm{k}_{1}\left(\mathrm{~d}_{1} w_{0}^{4}+2 \mathrm{k}_{1} w_{0}^{2}+2 \mathrm{k}_{3}\right)\left(8 \mathrm{~d}_{1} w_{0}^{4}+16 \mathrm{k}_{1} w_{0}^{2}+7 \mathrm{k}_{3}\right)+\mathrm{k}_{6}}{3 \mathrm{~d}_{3} \mathrm{k}_{3}\left(4 \mathrm{~d}_{1} w_{0}^{4}+8 \mathrm{k}_{1} w_{0}^{2}+5 \mathrm{k}_{3}\right)}\right), \\
& \mathrm{P}_{1}=\left(\frac{\mathrm{k}_{3} w_{0}}{8\left(\mathrm{~d}_{1} w_{0}^{4}+2 \mathrm{k}_{1} w_{0}^{2}+\mathrm{k}_{3}\right)}, \frac{\mathrm{k}_{6}}{16\left(\mathrm{~d}_{1} w_{0}^{4}+2 \mathrm{k}_{1} w_{0}^{2}+\mathrm{k}_{3}\right)}, y_{0}, \frac{\mathrm{k}_{3} w_{3}^{2}}{32 \mathrm{~d}_{3}\left(\mathrm{~d}_{1} w_{0}^{4}+2 \mathrm{k}_{1} w_{0}^{2}+\mathrm{k}_{3}\right)\left(\mathrm{d}_{1} w_{0}^{4}+2 \mathrm{k}_{1} w_{0}^{2}+2 \mathrm{k}_{3}\right)}\right), \\
& \mathrm{P}_{2}=\left(\frac{3 \mathrm{k}_{3} w_{0}}{8\left(\mathrm{~d}_{1} w_{0}^{4}+2 \mathrm{k}_{1} w_{0}^{2}+\mathrm{k}_{3}\right)}, \frac{3 \mathrm{k}_{3} w_{0}^{2}}{16\left(\mathrm{~d}_{1} w_{0}^{4}+2 \mathrm{k}_{1} w_{0}^{2}+\mathrm{k}_{3}\right)}, y_{0}, z_{2}\right),
\end{aligned}
$$

where

$$
z_{2}=\frac{\frac{3\left(8 c_{2} c_{3} k_{1} k_{3}^{2}+k_{6}\right)}{k_{3}\left(d_{1} w_{0}^{4}+2 k_{1} w_{0}^{2}+k_{3}\right)}+\frac{32\left(k_{6}-9 c_{2} c_{3} k_{1} k_{3}^{2}\right)}{k_{3}\left(4 d_{1} w_{0}^{4}+8 k_{1} w_{0}^{2}+5 k_{3}\right)}+192 c_{2} c_{3} k_{1}-\frac{11 k_{6}}{k_{3}\left(d_{1} w_{0}^{4}+2 k_{1} w_{0}^{2}+2 k_{3}\right)}}{96 d_{3}}>0 .
$$


Proof. The proof of i) and ii) is analogous to the given for Lemma 3.1. We only will prove claim iii). In the present case, since all the parameters are positive and the points of interest are in $\Gamma$, the equilibrium points for the differential system (1.1) must satisfy the system

$$
\begin{array}{r}
a_{1} R y+\left(b_{1}+w^{2}+x\right)(R(v-\rho)+(w+x) \rho)=0 \\
-x\left(d_{1}+a_{3} y\right)+w v=0 \\
\left(-a_{1} c_{1} w+\left(b_{1}+w^{2}+x\right)\left(d_{2}-a_{3} c_{2} x\right)\right)\left(b_{2}+y\right)+a_{2}\left(b_{1}+w^{2}+x\right) z=0 \\
-a_{2} c_{3} y+d_{3}\left(b_{2}+y\right)=0 .
\end{array}
$$

Assume that $P_{0}=\left(w_{0}, x_{0}, y_{0}, z_{0}\right) \in \Gamma$ is an equilibrium point satisfying system (4.1). Solving first, second, third, and fourth equation in the variables $a_{1}, a_{2}, a_{3}$, and $c_{1}$, respectively, one gets

$$
\begin{aligned}
& a_{1}=-\frac{\left(b_{1}+w_{0}^{2}+x_{0}\right)\left(R(v-\rho)+\rho\left(w_{0}+x_{0}\right)\right)}{R y_{0}}, a_{2}=\frac{d_{3}\left(b_{2}+y_{0}\right)}{c_{3} y_{0}}, a_{3}=\frac{v w_{0}-d_{1} x_{0}}{x_{0} y_{0}}, \\
& c_{1}=-\frac{R\left(c_{2} c_{3}\left(d_{1} x_{0}-v w_{0}\right)+c_{3} d_{2} y_{0}+d_{3} z_{0}\right)}{c_{3} w_{0}\left(v R+\rho\left(-R+w_{0}+x_{0}\right)\right)} .
\end{aligned}
$$

Taking $v=\frac{d_{1} x_{0}+k_{1}}{w_{0}}, d_{2}=\frac{c_{2} k_{1}}{y_{0}}, R=2\left(w_{0}+x_{0}\right)$ and $\rho=\frac{2 d_{1} x_{0}+2 k_{1}+k_{2}}{w_{0}}$, where $k_{1}, k_{2}>0$ we have that

$$
a_{1}=\frac{k_{2}\left(b_{1}+w_{0}^{2}+x_{0}\right)}{2 w_{0} y_{0}}, \quad a_{2}=\frac{d_{3}\left(b_{2}+y_{0}\right)}{c_{3} y_{0}}, \quad a_{3}=\frac{k_{1}}{x_{0} y_{0}}, \quad c_{1}=\frac{2 d_{3} z_{0}}{c_{3} k_{2}},
$$

and $P_{0} \in \Gamma$ is an equilibrium point for the differential system (1.1). Hence under these conditions for the parameters $a_{1}, a_{2}, a_{3}$, and $c_{1}$ the system (4.1) takes the form

$$
\begin{array}{r}
\left(\mathrm{b}_{1}+w^{2}+x\right)\left(k_{2}\left(w-2 w_{0}+x-2 x_{0}\right)+2 k_{1}\left(w-w_{0}+x-x_{0}\right)+2 d_{1}\left(w-w_{0}+x-x_{0}\right) x_{0}\right) \\
+\left(1 / y_{0}\right) k_{2}\left(w_{0}+x_{0}\right)\left(b_{1}+w_{0}^{2}+x_{0}\right) y=0 \\
-k_{1} w_{0} x y+x_{0}\left(k_{1} w-d_{1} w_{0} x+d_{1} w x_{0}\right) y_{0}=0 \\
\left(1 / x_{0}\right)\left(b_{1}+w^{2}+x\right)\left(-c_{2} c_{3} k_{1}\left(x-x_{0}\right)\left(b_{2}+y\right)+d_{3} x_{0}\left(b_{2}+y_{0}\right) z\right)-\left(1 / w_{0}\right) d_{3} w\left(b_{1}+w_{0}^{2}+x_{0}\right)\left(b_{2}+y\right) z_{0}=0 \\
b_{2} d_{3}\left(-y+y_{0}\right)=0 .
\end{array}
$$

Assume that $P_{1}=\left(w_{1}, x_{1}, y_{1}, z_{1}\right) \in \Gamma \backslash\left\{P_{0}\right\}$ satisfies (4.2). We will give conditions to find expressions for $P_{1}$. Since all the parameters are positive, from fourth equation we have $y_{1}=y_{0}$ and substituting $y_{1}$ in the other equations we have that system (4.2) simplifies to

$$
\begin{array}{r}
k_{2}\left(w_{0}+x_{0}\right)\left(b_{1}+w_{0}^{2}+x_{0}\right)+\left(b_{1}+w_{1}^{2}+x_{1}\right)\left(k_{2}\left(w_{1}-2 w_{0}+x_{1}-2 x_{0}\right)\right. \\
\left.+2 k_{1}\left(w_{1}-w_{0}+x_{1}-x_{0}\right)+2 d_{1}\left(w_{1}-w_{0}+x_{1}-x_{0}\right) x_{0}\right)=0, \\
w_{1} x_{0}-w_{0} x_{1}=0, \\
-w_{0}\left(b_{1}+w_{1}^{2}+x_{1}\right)\left(c_{2} c_{3} k_{1}\left(x_{1}-x_{0}\right)-d_{3} x_{0} z_{1}\right)-d_{3} w x_{0}\left(b_{1}+w_{0}^{2}+x_{0}\right) z_{0}=0 .
\end{array}
$$

From the third equation in (4.3) and solving for $z_{1}$ we have an expression in terms of $x_{1}$

$$
z_{1}=\frac{w_{1} z_{0}\left(b_{1}+w_{0}^{2}+x_{0}\right)}{w_{0}\left(b_{1}+w_{1}^{2}+x_{1}\right)}+\frac{c_{2} c_{3} k_{1}\left(x_{1}-x_{0}\right)}{d_{3} x_{0}}
$$

Now, from second equation $w_{1}=\frac{w_{0} x_{1}}{x_{0}}$. Substituting $w_{1}$ in first equation we have the equation

$$
\frac{1}{w_{0} x_{0}^{3}}\left(x_{1}-x_{0}\right)\left(w_{0}+x_{0}\right) \mathbf{S}_{0}=0
$$

where $\mathbf{S}_{0}$ is a quadratic polynomial in the variable $x_{1}$ :

$$
\mathbf{S}_{0}=w_{0}^{2}\left(2 k_{1}+k_{2}+2 d_{1} x_{0}\right) x_{1}^{2}+x_{0}\left(k_{2}\left(-w_{0}^{2}+x_{0}\right)+2 x_{0}\left(k_{1}+d_{1} x_{0}\right)\right) x_{1}
$$




$$
+x_{0}^{2}\left(-k_{2}\left(w_{0}^{2}+x_{0}\right)+b_{1}\left(2 k_{1}+k_{2}+2 d_{1} x_{0}\right)\right) .
$$

Suppose that $x_{1} \neq x_{0}$ and let $k_{3}>0$ such that $k_{2}\left(-w_{0}^{2}+x_{0}\right)+2 x_{0}\left(k_{1}+d_{1} x_{0}\right)=-k_{3}$ and $x_{0}=\frac{w_{0}^{2}}{2}$. Then, $k_{2}=2 k_{1}+\left(2 k_{3}\right) / w_{0}^{2}+d_{1} w_{0}^{2}$ and $\mathbf{S}_{0}$ simplifies to

$$
\begin{aligned}
\mathbf{S}_{0}= & (1 / 8)\left(4 \mathrm{~b}_{1} w_{0}^{2}\left(k_{3}+2 k_{1} w_{0}^{2}+\mathrm{d}_{1} w_{0}^{4}\right)-3\left(2 k_{3} w_{0}^{4}+2 k_{1} w_{0}^{6}+\mathrm{d}_{1} w_{0}^{8}\right)\right) \\
& -(1 / 2) \mathrm{k}_{3} w_{0}^{2} \mathrm{x}_{1}+2\left(\mathrm{k}_{3}+2 \mathrm{k}_{1} w_{0}^{2}+\mathrm{d}_{1} w_{0}^{4}\right) x_{1}^{2} .
\end{aligned}
$$

Let $k_{4}>0$ such that $4 b_{1} w_{0}^{2}\left(k_{3}+2 k_{1} w_{0}^{2}+d_{1} w_{0}^{4}\right)-3\left(2 k_{3} w_{0}^{4}+2 k_{1} w_{0}^{6}+d_{1} w_{0}^{8}\right)=k_{4}$, that is,

$$
b_{1}=\frac{3 d_{1} w_{0}^{8}+6 k_{1} w_{0}^{6}+6 k_{3} w_{0}^{4}+k_{4}}{4 d_{1} w_{0}^{6}+8 k_{1} w_{0}^{4}+4 k_{3} w_{0}^{2}}
$$

and equation (4.5) becomes the condition

$$
\mathbf{S}_{0}=\left(k_{4} / 8\right)-(1 / 2) k_{3} w_{0}^{2} x_{1}+2\left(k_{3}+2 k_{1} w_{0}^{2}+d_{1} w_{0}^{4}\right) x_{1}^{2}=0 .
$$

Solving $\mathbf{S}_{0}=0$ for $x_{1}$ we have two roots which without loss of generality will be labeled as

$$
x_{1,2}=\frac{k_{4}}{2 k_{3} w_{0}^{2} \pm 2 \sqrt{w_{0}^{4}\left(k_{3}^{2}-4 d_{1} k_{4}\right)-8 k_{1} k_{4} w_{0}^{2}-4 k_{3} k_{4}}} .
$$

Let $k_{5}>0$ such that $w_{0}^{4}\left(k_{3}^{2}-4 d_{1} k_{4}\right)-8 k_{1} k_{4} w_{0}^{2}-4 k_{3} k_{4}=k_{5}^{2}$. Taking $k_{5}=k_{3} w_{0}^{2} / 2$ we have $k_{4}=$ $\frac{3 k_{3}^{2} w_{0}^{4}}{16\left(\mathrm{~d}_{1} w_{0}^{4}+2 \mathrm{k}_{1} w_{0}^{2}+\mathrm{k}_{3}\right)}$ and

$$
x_{1}=\frac{k_{3} w_{0}^{2}}{16\left(d_{1} w_{0}^{4}+2 k_{1} w_{0}^{2}+k_{3}\right)}, \quad x_{2}=\frac{3 k_{3} w_{0}^{2}}{16\left(d_{1} w_{0}^{4}+2 k_{1} w_{0}^{2}+k_{3}\right)} .
$$

Since $w_{1}=\frac{w_{0} x_{1}}{x_{0}}$, we have that $w_{1}$ takes two values at $x_{1,2}$ given by $w_{1}=\frac{k_{3} w_{0}}{8\left(d_{1} w_{0}^{4}+2 k_{1} w_{0}^{2}+k_{3}\right)}$ and $w_{2}=$ $\frac{3 k_{3} w_{0}}{8\left(d_{1} w_{0}^{4}+2 k_{1} w_{0}^{2}+k_{3}\right)}$.

On the other hand, from (4.4) we have two values for $z_{1}$ given by

$$
\begin{aligned}
& z_{1}=\frac{3 \mathrm{~d}_{3} \mathrm{k}_{3} z_{0}\left(4 \mathrm{~d}_{1} w_{0}^{4}+8 \mathrm{k}_{1} w_{0}^{2}+5 \mathrm{k}_{3}\right)-4 \mathrm{c}_{2} \mathrm{c}_{3} \mathrm{k}_{1}\left(\mathrm{~d}_{1} w_{0}^{4}+2 \mathrm{k}_{1} w_{0}^{2}+2 \mathrm{k}_{3}\right)\left(8 \mathrm{~d}_{1} w_{0}^{4}+16 \mathrm{k}_{1} w_{0}^{2}+7 \mathrm{k}_{3}\right)}{32 \mathrm{~d}_{3}\left(\mathrm{~d}_{1} w_{0}^{4}+2 \mathrm{k}_{1} w_{0}^{2}+\mathrm{k}_{3}\right)\left(\mathrm{d}_{1} w_{0}^{4}+2 \mathrm{k}_{1} w_{0}^{2}+2 \mathrm{k}_{3}\right)}, \\
& z_{2}=\frac{3 \mathrm{~d}_{3} \mathrm{k}_{3} z_{0}\left(12 \mathrm{~d}_{1} w_{0}^{4}+24 \mathrm{k}_{1} w_{0}^{2}+13 \mathrm{k}_{3}\right)-4 \mathrm{c}_{2} \mathrm{c}_{3} \mathrm{k}_{1}\left(\mathrm{~d}_{1} w_{0}^{4}+2 \mathrm{k}_{1} w_{0}^{2}+2 \mathrm{k}_{3}\right)\left(8 \mathrm{~d}_{1} w_{0}^{4}+16 \mathrm{k}_{1} w_{0}^{2}+5 \mathrm{k}_{3}\right)}{32 \mathrm{~d}_{3}\left(\mathrm{~d}_{1} w_{0}^{4}+2 \mathrm{k}_{1} w_{0}^{2}+\mathrm{k}_{3}\right)\left(\mathrm{d}_{1} w_{0}^{4}+2 \mathrm{k}_{1} w_{0}^{2}+2 \mathrm{k}_{3}\right)} .
\end{aligned}
$$

Since $z_{1}$ must be positive, let $k_{6}>0$ such that

$$
3 \mathrm{~d}_{3} \mathrm{k}_{3} z_{0}\left(4 \mathrm{~d}_{1} w_{0}^{4}+8 \mathrm{k}_{1} w_{0}^{2}+5 \mathrm{k}_{3}\right)-4 \mathrm{c}_{2} \mathrm{c}_{3} \mathrm{k}_{1}\left(\mathrm{~d}_{1} w_{0}^{4}+2 \mathrm{k}_{1} w_{0}^{2}+2 \mathrm{k}_{3}\right)\left(8 \mathrm{~d}_{1} w_{0}^{4}+16 \mathrm{k}_{1} w_{0}^{2}+7 \mathrm{k}_{3}\right)=\mathrm{k}_{6}
$$

and hence solving this equation for $z_{0}$ we have

$$
z_{0}=\frac{4 c_{2} c_{3} k_{1}\left(d_{1} w_{0}^{4}+2 k_{1} w_{0}^{2}+2 k_{3}\right)\left(8 d_{1} w_{0}^{4}+16 k_{1} w_{0}^{2}+7 k_{3}\right)+k_{6}}{3 d_{3} k_{3}\left(4 d_{1} w_{0}^{4}+8 k_{1} w_{0}^{2}+5 k_{3}\right)} .
$$

Since $y_{1}=y_{0}$ we have obtained two points $P_{1}, P_{2} \in \Gamma \backslash\left\{P_{0}\right\}$ that are equilibrium points for system (1.1) and hence $P_{0}, P_{1}$ and $P_{2}$ satisfy the conditions as in claim iii) which completes the proof.

\subsection{Dynamics of one equilibrium point}

In this case we will consider that the hypothesis in Lemma $4.1 \mathrm{i}$ ) are valid. And that the linear approximation of the differential system (1.1) at $P_{0}$ is a one parameter matrix with respect to $d_{3}$, which we denote as $M_{0}\left(d_{3}\right)$. We will guarantee that a Hopf bifurcation takes place. 
Lemma 4.2. If the hypothesis of Lemma 4.1 i) are satisfied and

$$
\begin{aligned}
a_{1} & =\frac{(m+7) v}{4 b_{2}}, \quad a_{2}=\frac{2 d_{3}}{c_{3}}, \quad a_{3}=\frac{v}{2 b_{2}}, \quad b_{1}=2, \quad c_{1}=b_{2}, \quad c_{2}=\frac{b_{2}}{2}, \\
d_{1} & =\frac{v}{2}, \quad d_{2}=\frac{1}{16}(m+7) v, \quad R=\frac{32}{m+7}+4, \quad \rho=\frac{m+15}{8}, \text { and } \\
d_{31} & =\frac{(m+7)(29 m+7(\sqrt{m(25 m-354)+4489}-67)) v}{1024},
\end{aligned}
$$

where $m>0$, then the eigenvalues of the linear approximation $M_{0}\left(d_{31}\right)$ of system (1.1) at $P_{0}$ are

where

$$
\lambda_{1,2}= \pm i \sqrt{\mathcal{R}_{0}} v \text { and } \lambda_{3,4}=-\frac{1}{128}\left( \pm \sqrt{2} \sqrt{\mathcal{R}_{1}}+56\right) v,
$$

$$
\begin{aligned}
& \mathcal{R}_{0}=\frac{(m+7)(5 m+\sqrt{m(25 m-354)+4489}-45)}{1024}>0, \\
& \mathcal{R}_{1}=7(\sqrt{m(25 m-354)+4489}+157)+m(-5 m+\sqrt{m(25 m-354)+4489}-102) .
\end{aligned}
$$

We have that

$$
\mathcal{R}_{1}>0 \text { if } \mathrm{m}<\mathrm{m}_{0}:=14.7403 ; \mathcal{R}_{1}=0 \text { if } \mathrm{m}=\mathrm{m}_{0} ; \text { and } \mathcal{R}_{1}<0 \text { if } \mathrm{m}>\mathrm{m}_{0} \text {. }
$$

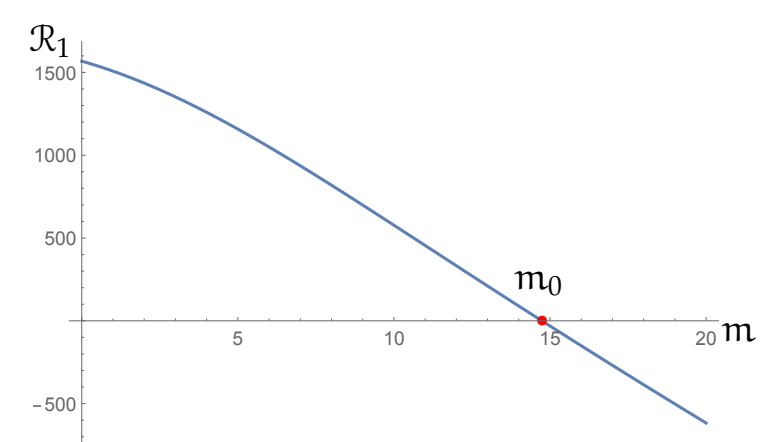

Figure 2: Graphic of $\mathcal{R}_{1}(\mathrm{~m})$.

Proof. We assume the hypothesis as in Lemma $4.1 \mathrm{i}$ ) are valid. In this occasion we set $a_{3}=\frac{d_{1} k_{1}}{b_{2} d_{3}}, k_{4}=k_{1}^{2} v^{2}$, $d_{1}=\frac{v}{2}$ and $k_{1}=d_{3}$. Then we have that the linear approximation $M_{0}\left(d_{3}\right)$ has characteristic polynomial

$$
\operatorname{pol}_{0}\left(\lambda, d_{3}\right)=\lambda^{4}+A_{10} \lambda^{3}+A_{20} \lambda^{2}+A_{30} \lambda+A_{40},
$$

where

$$
\begin{aligned}
& A_{10}=v-\frac{c_{2} v}{4 b_{2}}, \quad A_{20}=\frac{a_{1}^{2} b_{2}^{2} c_{1}+a_{1} b_{2} v\left(2 b_{2}-c_{1}\right)+8 c_{2} d_{3} v}{32 b_{2}} \\
& A_{30}=\frac{v\left(3 a_{1}^{2} b_{2}^{2} c_{1}+28 a_{1} b_{2} c_{2} v+64 c_{2} d_{3} v\right)}{256 b_{2}} \text { and } A_{40}=\frac{1}{64} a_{1} c_{2} d_{3} v^{2} .
\end{aligned}
$$

In this case, we have that $(2.1)$ is $\mathbf{E Q}=\frac{v^{2} \mathbf{F}_{0}}{65536 \mathrm{~b}_{2}^{3}}$, where

$$
\begin{aligned}
F_{0}= & b_{2}\left(3 a_{1}^{2} b_{2}^{2} c_{1}+28 a_{1} b_{2} c_{2} v+64 c_{2} d_{3} v\right)^{2}-2\left(4 b_{2}-c_{2}\right) \times\left(a_{1}^{2} b_{2}^{2} c_{1}+a_{1} b_{2} v\left(2 b_{2}-c_{1}\right)+8 c_{2} d_{3} v\right) \\
& \times\left(3 a_{1}^{2} b_{2}^{2} c_{1}+28 a_{1} b_{2} c_{2} v+64 c_{2} d_{3} v\right)+64 a_{1} b_{2} c_{2} d_{3} v^{2}\left(c_{2}-4 b_{2}\right)^{2} .
\end{aligned}
$$


Make $c_{2}=\frac{b_{2}}{2}, c_{1}=b_{2}, a_{1}=\frac{k_{5}+7 v}{4 b_{2}}$, and $k_{5}=m v$, where $m$ is a positive real number. Then, we have that $\mathbf{E Q}\left(d_{31}\right)=0$, where

$$
d_{31}=\frac{(m+7)(29 m+7(\sqrt{m(25 m-354)+4489}-67)) v}{1024}>0
$$

and all the coefficients of $\operatorname{pol}_{0}\left(\lambda, d_{31}\right)$ are positive. The roots of the characteristic polynomial are obtained from Lemma 2.1 (ii) and a direct calculation shows that $\mathcal{R}_{1}$ satisfies the claimed properties (see Fig. 2).

Lemma 4.3. If the hypothesis of Lemma 4.2 are satisfied and $m=\frac{354}{25}$, then the first Lyapunov coefficient for the system (1.1) at $\mathrm{P}_{0}$ is negative. Moreover, the transversality condition holds: $\frac{\partial \operatorname{Re}\left(\lambda_{1,2}\right)}{\partial \mathrm{d}_{3}}\left(\mathrm{~d}_{31}\right) \neq 0$.

Proof. Under the hypothesis of Lemma 4.2 if $m=\frac{354}{25}$, we have that

$$
\frac{\partial \operatorname{Re}\left(\lambda_{1,2}\right)}{\partial d_{3}}\left(d_{31}\right)=\frac{10720000}{2218824689} \neq 0
$$

and that the first Lyapunov coefficient is $\ell_{1}\left(P_{0}\right)=-\frac{\mathcal{N}_{0}}{\mathcal{N}_{1}}<0$, where

$$
\begin{aligned}
& \mathcal{N}_{0}=18789363664718319688470457812986851 \sqrt{\frac{29}{5}}, \\
& \mathcal{N}_{1}=9534260680917181346060000\left(18351686591+766720 b_{2}^{2}\left(15341+125 c_{3}^{2}\right)\right) .
\end{aligned}
$$

Next theorem follows from Lemma 4.3 and the Andronov-Hopf Theorem.

Theorem 4.4 ( $f_{1}$ Holling IV, one equilibrium point). Assume that the parameters of system (1.1) satisfy the hypothesis of Lemma 4.3. Then, the system exhibits a supercritical Hopf bifurcation at $\mathrm{P}_{0}$ with respect to the parameter $\mathrm{d}_{3}$ and its bifurcation value is $\mathrm{d}_{31}$.

\subsection{Dynamics of two equilibrium points}

If the hypothesis of Lemma 4.1 ii) hold and $k_{1}=d_{1} w_{0}^{2}$ and $k_{3}=d_{1} w_{0}^{4}$, then a direct calculation shows that the linear approximation for the system (1.1) at $\mathrm{P}_{1}$ has a characteristic polynomial with constant term equals to zero. Hence, from the Routh-Hurwitz test we have that in these conditions $P_{1}$ is locally unstable.

Now, we will consider the linear approximation of system (1.1) at $P_{0}$ as a one parameter matrix with respect to $b_{2}$, which we denote as $M_{0}\left(b_{2}\right)$.

Lemma 4.5. If the hypothesis of Lemma 4.1 ii) are satisfied and

$$
\begin{aligned}
& a_{1}=\frac{92015625 d_{3}}{246208 y_{0}}, \\
& a_{2}=\frac{d_{3}\left(b_{2}+y_{0}\right)}{c_{3} y_{0}}, \\
& a_{3}=\frac{58890 d_{3}}{3847 y_{0}}, \\
& \mathrm{~b}_{1}=\frac{241}{64} \text {, } \\
& c_{1}=\frac{351\left(b_{2}+y_{0}\right)}{2500} \text {, } \\
& c_{2}=\frac{351\left(b_{2}+y_{0}\right)}{6040}, \\
& \mathrm{~d}_{1}=\frac{29445 \mathrm{~d}_{3}}{3847}, \\
& d_{2}=\frac{13689 d_{3}\left(b_{2}+y_{0}\right)}{7694 y_{0}} \text {, } \\
& \mathrm{R}=8, \\
& \rho=\frac{471120 d_{3}}{3847}, \\
& \mathrm{~b}_{20}=\frac{95480385736 \mathrm{y}_{0}}{16391886457} \text {, }
\end{aligned}
$$

then the eigenvalues of the linear approximation $\mathrm{M}_{0}\left(\mathrm{~b}_{20}\right)$ of system (1.1) at $\mathrm{P}_{0}$ are

$$
\lambda_{1,2}= \pm i 353340 \sqrt{\frac{21305}{63059587200079}} d_{3} \text { and } \lambda_{3,4}=-\frac{117(94375 \mp i \sqrt{194109621935}) d_{3}}{1923500} .
$$

Proof. Suppose that the parameters of the system (1.1) satisfy the hypothesis as in Lemma 4.1 ii). As was made in the other cases, setting $k_{1}=d_{1} w_{0}^{2}$ and $k_{3}=d_{1} w_{0}^{4}$, the linear approximation $M_{0}\left(b_{2}\right)$ has 
characteristic polynomial $\operatorname{pol}_{0}\left(\lambda, b_{2}\right)=\lambda^{4}+B_{1} \lambda^{3}+B_{2} \lambda^{2}+B_{3} \lambda+B_{4}$, such that

$$
\begin{aligned}
& B_{1}=\frac{1}{125} d_{1}\left(-\frac{755 c_{2} w_{0}^{2}}{b_{2}+y_{0}}-256 w_{0}+\frac{125\left(11 w_{0}+6\right)}{w_{0}+2}\right), \quad B_{2}=\frac{d_{1} w_{0}\left(\mathcal{K}_{0}\right)}{31250\left(w_{0}+2\right) y_{0}\left(b_{2}+y_{0}\right)}, \\
& B_{3}=-\frac{c_{2} d_{1}^{2} w_{0}^{2}\left(\mathcal{K}_{1}\right)}{15625\left(w_{0}+2\right) y_{0}\left(b_{2}+y_{0}\right)}, \quad B_{4}=\frac{16308 b_{2} c_{2} d_{1}^{3} d_{3} w_{0}^{3}}{625 y_{0}\left(b_{2}+y_{0}\right)}
\end{aligned}
$$

where

$$
\begin{aligned}
\mathcal{K}_{0}= & b_{2}\left(w_{0}+2\right)\left(c_{2} w_{0}\left(85315 d_{1} w_{0}-14812 d_{1}+188750 d_{3}\right)+135000 d_{1} y_{0}\right) \\
& +d_{1} y_{0}\left(c_{2} w_{0}\left(w_{0}\left(471875 w_{0}-1147312\right)-1162124\right)+135000\left(w_{0}+2\right) y_{0}\right), \\
\mathcal{K}_{1}= & b_{2}\left(16 d_{1} w_{0}\left(229 w_{0}-12902\right)+755 d_{3}\left(w_{0}\left(256 w_{0}-863\right)-750\right)\right)+4 d_{1} w_{0}\left(102841 w_{0}+152242\right) y_{0} .
\end{aligned}
$$

Setting $w_{0}=2, c_{2}=\frac{351\left(b_{2}+y_{0}\right)}{6040}$ and $d_{1}=\frac{29445 d_{3}}{3847}$, we have that $(2.1)$ is

$$
E Q=\frac{1092211792881596337270411 d_{3}^{6}\left(95480385736 y_{0}-16391886457 b_{2}\right)}{2532346607490523417913281250 y_{0}}
$$

Moreover, all the coefficients of $\operatorname{pol}_{0}\left(\lambda, b_{20}\right)$ are positive and $\mathbf{E Q}\left(b_{20}\right)=0$, where $b_{20}=\frac{95480385736 y_{0}}{16391886457}$. Therefore, the proof is concluded using Lemma $2.1 \mathrm{ii}$ ).

Lemma 4.6. If the hypothesis of Lemma 4.5 are satisfied, then the first Lyapunov coefficient $\ell_{1}\left(\mathrm{P}_{0}, \mathrm{~b}_{20}\right)$ for the system (1.1) at $\mathrm{P}_{0}$ is negative. Moreover, the transversality condition holds: $\frac{\partial \operatorname{Re}\left(\lambda_{1,2}\right)}{\partial \mathrm{b}_{2}}\left(\mathrm{~b}_{20}\right) \neq 0$.

Proof. Under the hypothesis of Lemma 4.5 we have that

$$
\frac{\partial \operatorname{Re}\left(\lambda_{1,2}\right)}{\partial b_{2}}\left(b_{20}\right)=-\frac{11801569339356166931213599722872813535 d_{3}}{19478350806921655804860578959172649472 y_{0}} \neq 0
$$

and that the first Lyapunov coefficient is $\ell_{1}\left(P_{0}, b_{20}\right)=-\frac{\mathcal{M T}_{0}}{\mathcal{M}_{1}}<0$, where

$$
\begin{aligned}
\mathcal{M}_{0}= & 217676423201567543587668555270113809421753406988141195772466 \\
& 9764374472301079117588144055366058034338623801083047117711125 \sqrt{\frac{315297936000395}{4261},} \\
\mathcal{M}_{1}= & 6071506970287455575459137144567343632577578601721847016911101682580953931726107867136 \\
& \times\left(2409398303445653547654\left(457623738857453785461 c_{3}^{2}+4926530250006171875\right) y_{0}^{2}\right. \\
& +222238726372030874554317307928114420046875) .
\end{aligned}
$$

Next theorem follows from Lemma 4.6 and the Andronov-Hopf Theorem.

Theorem 4.7 ( $f_{1}$ Holling IV, two equilibrium points). Assume that the parameters of system (1.1) satisfy the hypothesis of Lemma 4.6. Then, the system exhibits a supercritical Hopf bifurcation at $\mathrm{P}_{0}$ with respect to the parameter $\mathrm{b}_{2}$ and its bifurcation value is $\mathrm{b}_{20}$.

\subsection{Dynamics of three equilibrium points}

If the hypothesis of Lemma 4.1 iii) hold and $k_{1}=d_{1} w_{0}^{2}$ and $k_{3}=d_{1} w_{0}^{4}$, then a direct calculation shows that the linear approximation for the system (1.1) at $\mathrm{P}_{2}$ has a characteristic polynomial with a negative constant term. Hence, from Routh-Hurwitz test we have that under these conditions the equilibrium point $P_{2}$ is locally unstable.

This time we will consider the linear approximations of system (1.1) at $P_{0}$ and $P_{1}$ as one parameter matrices with respect to $b_{2}$, which we denote as $M_{0}\left(b_{2}\right)$ and $M_{1}\left(b_{2}\right)$, respectively. 
Lemma 4.8. Assume that the hypothesis of Lemma 4.1 iii) are satisfied.

a) If

$$
\begin{aligned}
\mathrm{a}_{1} & =\frac{819909405 \mathrm{~d}_{3}}{45928192 \mathrm{y}_{0}}, \quad \mathrm{a}_{2}=\frac{\mathrm{d}_{3}\left(\mathrm{~b}_{2}+\mathrm{y}_{0}\right)}{\mathrm{c}_{3} \mathrm{y}_{0}}, \quad \mathrm{a}_{3}=\frac{65619 \mathrm{~d}_{3}}{717628 \mathrm{y}_{0}}, \mathrm{~b}_{1}=\frac{963}{64}, \quad \mathrm{c}_{1}=\frac{317\left(\mathrm{~b}_{2}+\mathrm{y}_{0}\right)}{333200}, \\
\mathrm{c}_{2} & =\frac{317\left(\mathrm{~b}_{2}+\mathrm{y}_{0}\right)}{1622880}, \mathrm{~d}_{1}=\frac{65619 \mathrm{~d}_{3}}{1435256}, \mathrm{~d}_{2}=\frac{100489 \mathrm{~d}_{3}\left(\mathrm{~b}_{2}+\mathrm{y}_{0}\right)}{703275440 \mathrm{y}_{0}}, \mathrm{R}=24, \rho=\frac{262476 \mathrm{~d}_{3}}{179407}, \text { and } \\
\mathrm{b}_{20} & =\frac{13416348622408722 \mathrm{y}_{0}}{582645121928723},
\end{aligned}
$$

then $\mathrm{P}_{1}$ is locally stable and the eigenvalues of the linear approximation $\mathrm{M}_{0}\left(\mathrm{~b}_{20}\right)$ of system (1.1) at $\mathrm{P}_{0}$ are

$$
\lambda_{1,2}= \pm i 393714 \sqrt{\frac{19316813}{104530613389866407261}} d_{3} \text { and } \lambda_{3,4}=-\frac{951(57477 \mp i \sqrt{31910155004111}) d_{3}}{23911364960} .
$$

b) If

$$
\begin{aligned}
& \mathrm{a}_{1}=\frac{326483386887 \mathrm{~d}_{3}}{29814521554138240 \mathrm{y}_{0}}, \quad \mathrm{a}_{2}=\frac{\mathrm{d}_{3}\left(\mathrm{~b}_{2}+\mathrm{y}_{0}\right)}{\mathrm{c}_{3} \mathrm{y}_{0}}, \quad \mathrm{a}_{3}=\frac{7617820000 \mathrm{~d}_{3}}{46585189928341 \mathrm{y}_{0}}, \quad \mathrm{~b}_{1}=\frac{47187}{640000}, \\
& \mathrm{c}_{1}=\frac{5441300\left(\mathrm{~b}_{2}+\mathrm{y}_{0}\right)}{332367}, \quad \mathrm{c}_{2}=\frac{13603250\left(\mathrm{~b}_{2}+\mathrm{y}_{0}\right)}{4047057}, \mathrm{~d}_{1}=\frac{3808910000 \mathrm{~d}_{3}}{46585189928341}, \mathrm{~d}_{2}=\frac{11843098276000 \mathrm{~d}_{3}\left(\mathrm{~b}_{2}+\mathrm{y}_{0}\right)}{549658655964495459 \mathrm{y}_{0}}, \\
& \mathrm{R}=\frac{399}{625}, \rho=\frac{8531958400 \mathrm{~d}_{3}}{46585189928341}, \text { and } \mathrm{b}_{21}=\frac{1283288513820972429108420117140000 \mathrm{y}_{0}}{1003762066972593392736885855153},
\end{aligned}
$$

then $\mathrm{P}_{0}$ is locally unstable and the eigenvalues of the linear approximation $\mathrm{M}_{1}\left(\mathrm{~b}_{21}\right)$ of system (1.1) at $\mathrm{P}_{1}$ are

$$
\begin{aligned}
& \lambda_{1,2}= \pm i 761782000 \sqrt{\frac{1473121548605}{18186082630409313413515160609495761}} d_{3}, \\
& \lambda_{3,4}=-\frac{272065(+210492409575823459 \mp \sqrt{44189378141813568890621430192477481}) d_{3}}{462833171939453734903948632} .
\end{aligned}
$$

Proof. Suppose that the parameters of the system (1.1) satisfy the hypothesis as in Lemma 4.1 iii). To simplify the calculations set $k_{1}=\mathrm{d}_{1} w_{0}^{2}$ and $k_{3}=\mathrm{d}_{1} w_{0}^{4}$.

We prove claim a).

The linear approximation $M_{0}\left(b_{2}\right)$ has characteristic polynomial $\operatorname{pol}_{0}\left(\lambda, b_{2}\right)=\lambda^{4}+B_{01} \lambda^{3}+B_{02} \lambda^{2}+$ $\mathrm{B}_{03} \lambda+\mathrm{B}_{04}$, such that

$$
\begin{aligned}
\mathrm{B}_{01} & =\mathrm{d}_{1}\left(-\frac{5120 w_{0}}{2499}-\frac{16}{w_{0}+2}+11\right)-\frac{620 \mathrm{c}_{2} \mathrm{c}_{3} \mathrm{~d}_{1}^{3} w_{0}^{10}+\mathrm{k}_{6}}{51 \mathrm{c}_{3} \mathrm{~d}_{1}^{2} w_{0}^{8}\left(\mathrm{~b}_{2}+\mathrm{y}_{0}\right)}, \mathrm{B}_{02}=\frac{s_{0}}{254898 \mathrm{c}_{3} \mathrm{~d}_{1}^{2} w_{0}^{8}\left(w_{0}+2\right) y_{0}\left(\mathrm{~b}_{2}+y_{0}\right)}, \\
\mathrm{B}_{03} & =\frac{s_{1}}{254898 \mathrm{c}_{3} \mathrm{~d}_{1} w_{0}^{8}\left(w_{0}+2\right) y_{0}\left(\mathrm{~b}_{2}+y_{0}\right)}, \quad \mathrm{B}_{04}=\frac{3596 \mathrm{~b}_{2} \mathrm{~d}_{3}\left(620 \mathrm{c}_{2} \mathrm{c}_{3} \mathrm{~d}_{1}^{3} w_{0}^{10}+\mathrm{k}_{6}\right)}{42483 \mathrm{c}_{3} w_{0}^{7} \mathrm{y}_{0}\left(\mathrm{~b}_{2}+\mathrm{y}_{0}\right)},
\end{aligned}
$$

where

$$
\begin{aligned}
s_{0}= & b_{2}\left(w_{0}+2\right)\left(4 c_{3} d_{1}^{4} w_{0}^{9}\left(c_{2} w_{0}\left(349525 w_{0}-189991\right)+275094 y_{0}\right)+3098760 c_{2} c_{3} d_{1}^{3} d_{3} w_{0}^{10}+d_{1} k_{6}\left(2255 w_{0}-2048\right)+4998 d_{3} k_{6}\right) \\
& +d_{1} y_{0}\left(4 c_{3} d_{1}^{3} w_{0}^{9}\left(c_{2} w_{0}\left(w_{0}\left(1936725 w_{0}-4838131\right)-5028122\right)+275094\left(w_{0}+2\right) y_{0}\right)+k_{6}\left(w_{0}\left(12495 w_{0}-32036\right)-34084\right)\right), \\
s_{1}= & b_{2}\left(-2 d_{3}\left(w_{0}\left(5120 w_{0}-17249\right)-14994\right)\left(620 c_{2} c_{3} d_{1}^{3} w_{0}^{10}+k_{6}\right)\right. \\
& \left.-d_{1} w_{0}\left(c_{2} c_{3} d_{1}^{3}\left(999565 w_{0}-868998\right) w_{0}^{10}+k_{6}\left(3011 w_{0}+7974\right)\right)\right) \\
& -d_{1} w_{0} y_{0}\left(c_{2} c_{3} d_{1}^{3}\left(14376685 w_{0}+25885242\right) w_{0}^{10}+k_{6}\left(24587 w_{0}+51126\right)\right) .
\end{aligned}
$$

Setting $k_{6}=c_{2} c_{3} d_{1}^{3} w_{0}^{10}, w_{0}=4, k_{7}=\frac{1}{10}, c_{2}=\frac{317\left(b_{2}+y_{0}\right)}{1622880}$, and $d_{1}=\frac{65619 d_{3}}{1435256}$, it follows that (2.1) for $P_{0}$ becomes

$$
\mathbf{E Q}_{\mathrm{P}_{0}}=\frac{590603773376433329361449973 \mathrm{~d}_{3}^{6}\left(13416348622408722 \mathrm{y}_{0}-582645121928723 \mathrm{~b}_{2}\right)}{526097508598209708322692022112329253539418243072000 \mathrm{y}_{0}} .
$$


Moreover, all the coefficients of $\operatorname{pol}_{0}\left(\lambda, b_{20}\right)$ are positive and $\mathbf{E Q}_{\mathrm{P}_{0}}\left(\mathrm{~b}_{20}\right)=0$, where $\mathrm{b}_{20}=\frac{13416348622408722 \mathrm{y}_{0}}{582645121928723}$. Therefore, the proof of the claim for $\mathrm{P}_{0}$ is concluded using Lemma 2.1 (ii). Now, we prove the claim for $P_{1}$. Under these assignations as above a calculation shows that the characteristic polinomial $\operatorname{pol}_{1}(\lambda)$ of $M_{1}\left(b_{2}\right)$ has positive coefficients and that (2.1) for $P_{1}$ is

$$
\mathbf{E Q}_{\mathrm{P}_{1}}=-\frac{22195448555703486537 \mathrm{~d}_{3}^{6} \partial_{0}}{36987799937559506249199370394982860028164827198259200000000 y_{0}^{2}}<0,
$$

where

$$
\begin{aligned}
J_{0}= & 17776155316167905958812163517 b_{2}^{2}+1095685583423619310437058086372 b_{2} y_{0} \\
& +1077907578968001990869491705056 y_{0}^{2} .
\end{aligned}
$$

Hence, from Lemma 2.1 (i) $\mathrm{P}_{1}$ is locally asymptotically stable.

We now prove claim $b$ ).

The linear approximation $M_{1}\left(b_{2}\right)$ has characteristic polynomial $\operatorname{pol}_{1}\left(\lambda, b_{2}\right)=\lambda^{4}+B_{11} \lambda^{3}+B_{12} \lambda^{2}+$ $\mathrm{B}_{13} \lambda+\mathrm{B}_{14}$, such that

where

$$
\begin{aligned}
& \mathrm{B}_{11}=\mathrm{d}_{1}\left(-\frac{51 w_{0}}{3920}-\frac{1}{2\left(w_{0}+2\right)}+\frac{13}{4}\right)-\frac{\mathrm{k}_{6}}{640 \mathrm{c}_{3} \mathrm{~d}_{1}^{2} w_{0}^{8}\left(\mathrm{~b}_{2}+y_{0}\right)}, \quad \mathrm{B}_{12}=\frac{\mathcal{D}_{0}}{2508800 \mathrm{c}_{3} \mathrm{~d}_{1}^{2} w_{0}^{8}\left(w_{0}+2\right) y_{0}\left(\mathrm{~b}_{2}+y_{0}\right)}, \\
& \mathrm{B}_{13}=\frac{\mathcal{D}_{1}}{2508800 \mathrm{c}_{3} \mathrm{~d}_{1} w_{0}^{8}\left(w_{0}+2\right) \mathrm{y}_{0}\left(\mathrm{~b}_{2}+y_{0}\right)}, \text { and } \quad \mathrm{B}_{14}=\frac{93 \mathrm{~b}_{2} \mathrm{~d}_{3} \mathrm{k}_{6}}{2508800 \mathrm{c}_{3} w_{0}^{7} \mathrm{y}_{0}\left(\mathrm{~b}_{2}+y_{0}\right)^{\prime}},
\end{aligned}
$$

$$
\begin{aligned}
\mathcal{D}_{0}= & b_{2}\left(w_{0}+2\right)\left(20 c_{3} d_{1}^{4} w_{0}^{9}\left(c_{2} w_{0}\left(773109 w_{0}+3872\right)+2976 y_{0}\right)+d_{1} k_{6}\left(24939 w_{0}-128\right)+3920 d_{3} k_{6}\right) \\
& +2 d_{1} y_{0}\left(10 c_{3} d_{1}^{3}\left(w_{0}+2\right) w_{0}^{9}\left(c_{2} w_{0}\left(773109 w_{0}+3872\right)+2976 y_{0}\right)+k_{6}\left(w_{0}\left(12495 w_{0}+18556\right)-11888\right)\right), \\
\mathcal{D}_{1}= & b_{2}\left(d_{1} w_{0}\left(20 c_{2} c_{3} d_{1}^{3}\left(2351229 w_{0}+4764062\right) w_{0}^{10}+k_{6}\left(73431 w_{0}+148786\right)\right)+d_{3} k_{6}\left(w_{0}\left(12638-51 w_{0}\right)+23520\right)\right) \\
& +2 d_{1} w_{0} y_{0}\left(10 c_{2} c_{3} d_{1}^{3}\left(2351229 w_{0}+4764062\right) w_{0}^{10}+k_{6}\left(36669 w_{0}+74300\right)\right) .
\end{aligned}
$$

Setting $k_{6}=c_{2} c_{3} d_{1}^{3} w_{0}^{10}, w_{0}=\frac{28}{100}, k_{7}=\frac{2}{10}, c_{2}=\frac{13603250\left(b_{2}+y_{0}\right)}{4047057}$ and $d_{1}=\frac{3808910000 d_{3}}{46585189928341}$, we have that equation (2.1) is

$$
\begin{aligned}
\mathbf{E Q}_{\mathrm{P}_{1}}= & \frac{\mathcal{D}_{1}}{91868984290256955917703199862023417243228416635468310974676089991421968882194679209837255865548448 \mathrm{y}_{0}}, \\
\mathcal{D}_{1}= & 551669191500464941759890880349889453125 \mathrm{~d}_{3}^{6}\left(1003762066972593392736885855153 \mathrm{~b}_{2}\right. \\
& \left.-1283288513820972429108420117140000 \mathrm{y}_{0}\right) .
\end{aligned}
$$

Moreover, all the coefficients of $\operatorname{pol}_{1}\left(\lambda, b_{21}\right)$ are positive and $\mathbf{E} \mathbf{Q}_{\mathbf{P}_{1}}\left(b_{21}\right)=0$, where

$$
\mathrm{b}_{21}=\frac{1283288513820972429108420117140000 \mathrm{y}_{0}}{1003762066972593392736885855153} .
$$

Therefore, the proof is concluded by using Lemma 2.1 ii). Finally, under these parameter conditions a calculation shows that the characteristic polynomial $\operatorname{pol}_{0}\left(\lambda, b_{21}\right)$ has positive coefficients and $\mathbf{E Q}_{\mathrm{P}_{0}}\left(\mathrm{~b}_{21}\right)>$ 0 . Hence claim for $\mathrm{P}_{0}$ follows from Lemma $2.1 \mathrm{i}$ ).

\section{Lemma 4.9.}

i) If the hypothesis of Lemma 4.8 a) are satisfied, then the first Lyapunov coefficient $\ell_{1}\left(\mathrm{P}_{0}, \mathrm{~b}_{20}\right)$ for the system (1.1) at $\mathrm{P}_{0}$ is negative. Moreover, the transversality condition holds: $\frac{\partial \operatorname{Re}\left(\lambda_{1,2}\right)}{\partial \mathrm{b}_{2}}\left(\mathrm{~b}_{20}\right) \neq 0$.

ii) If the hypothesis of Lemma $4.8 \mathrm{~b})$ are satisfied, then the first Lyapunov coefficient $\ell_{1}\left(\mathrm{P}_{1}, \mathrm{~b}_{21}\right)$ for the system (1.1) at $\mathrm{P}_{1}$ is negative. Moreover, the transversality condition holds: $\frac{\partial \operatorname{Re}\left(\lambda_{1,2}\right)}{\partial \mathrm{b}_{2}}\left(\mathrm{~b}_{21}\right) \neq 0$. 
Proof. Proof of claim i): Under the hypothesis of Lemma 4.8 a) we have that

$$
\frac{\partial \operatorname{Re}\left(\lambda_{1,2}\right)}{\partial \mathrm{b}_{2}}\left(\mathrm{~b}_{20}\right)=-\frac{83966322790024614390702183754882228526848866556509 \mathrm{~d}_{3}}{560365662081392491481014219333781446381362348589949294 \mathrm{y}_{0}} \neq 0
$$

and that the first Lyapunov coefficient is $\ell_{1}\left(P_{0}, b_{20}\right)=-\frac{\mathcal{H}_{0}}{\mathcal{H}_{1}}<0$, where

$$
\begin{aligned}
\mathcal{H}_{0}= & 65108103224918914363893060962780 \sqrt{(58833390947229894479548164019310703020583084463339583499808327} \\
& 7521455881344535933813014375028123233745774466401515794984655051823072 \\
& 9424619846403866903862305677316411299296215210484026129430957410007553 \\
& 2702430128458655852906518055999193476399240810745150112683711440889205 \\
& 645543737105859057067802577242101 / 19316813), \\
\mathcal{H}_{1}= & 135750239588722107527224073922273432429534300327621012960131383507859631752974912752548439494 \\
& 1032826238376353233980675799(149014220778833149749810123716943909346072211821852591354202912 \\
& \left.+70372176667364736659077623\left(417323813582325947581870707654400+23383467340027183581879286644543 c_{3}^{2}\right) y_{0}^{2}\right) .
\end{aligned}
$$

Proof of claim ii): Under the hypothesis of Lemma $4.8 \mathrm{~b})$ we have that $\frac{\partial \operatorname{Re}\left(\lambda_{1,2}\right)}{\partial \mathrm{b}_{2}}\left(\mathrm{~b}_{21}\right)=\frac{\mathcal{T}_{0}}{\mathcal{T}_{1}} \neq 0$, where

$\mathcal{T}_{0}=47671642942992449797220740874336686735021383216432164461984587938993170270841661065492697 \mathrm{~d}_{3}$,

$\mathcal{T}_{1}=8660650070812536285392147610785201852358859609440080367793759296208919851350789498468218223447310664565380 y_{0}$,

and that the first Lyapunov coefficient is $\ell_{1}\left(P_{1}, b_{21}\right)=-\frac{\mathcal{L}_{0}}{\mathcal{L}_{1}}<0$, where

$$
\begin{aligned}
\mathcal{L}_{0}= & 6503722355183270578986766038515916230112374732311207160516265784528904 \\
& 3153320886782792177614429546205729992837090314159291751234486162303263 \\
& 46564390135160762444599512292579159910216834713481092680382200019744055 \\
& 74324862876235808455946403420610582397043771383526920963463168628907547 \\
& 106947691435788481666011312625306834124000000 \\
& \times \sqrt{(636512892064325969473030621332351635 / 42089187103),} \\
\mathcal{L}_{1}= & 1238643346392328912044675932710950343631164158700044380545912113846936 \\
& 0791269184611969358195576410247489562144022760515809253707052505638169 \\
& 7947297683537158372905436825837200512339727096499853148746706661906624 \\
& 7656923(4345553384671872346235422851807062198737763810095857262301925 \\
& 1110349971499132513730659984821588845514436143324311469 \\
& +84144203552867157412129081289671638443958865502261810000000 \\
& \times(14528750538623337057293427186537423079390182027665096772538931652 \\
& \left.\left.+572583807522980563175352059472333142429712816870609580978125 c_{3}^{2}\right) y_{0}^{2}\right) .
\end{aligned}
$$

Next theorem follows from Lemma 4.9 and the Andronov-Hopf Theorem.

Theorem 4.10 ( $f_{1}$ Holling IV, three equilibrium points).

i) Assume that the parameters of system (1.1) satisfy the hypothesis of Lemma 4.8 a). Then, $\mathrm{P}_{1}$ is locally stable and the system exhibits a supercritical Hopf bifurcation at $\mathrm{P}_{0}$ with respect to the parameter $\mathrm{b}_{2}$ and its bifurcation value is $\mathrm{b}_{20}$.

ii) Assume that the parameters of system (1.1) satisfy the hypothesis of Lemma $4.8 \mathrm{~b}$ ). Then, $\mathrm{P}_{0}$ is locally unstable and the system exhibits a supercritical Hopf bifurcation at $\mathrm{P}_{1}$ with respect to the parameter $\mathrm{b}_{2}$ and its bifurcation value is $b_{21}$. 


\section{Numerical examples}

In all cases, the coexistence of the three species takes place due to the existence of a supercritical Hopf bifurcation with respect to the corresponding parameters. A direct calculation shows that the hypothesis of the proved theorems for the differential system (1.1) are valid for parameters values with ecological sense, we show this in the following numerical examples.

\section{1. $\mathrm{f}_{1}$ Holling $I I$}

In the following two examples the functional response $f_{1}$ in the system (1.1) is Holling type II.

Example 5.1. From the hypothesis in Theorem 3.4 the differential system (1.1) takes the form

$$
\begin{array}{ll}
\dot{w}=\frac{1}{37} v w\left(-\frac{37 y}{b_{2}(w+x+1)}-2 w-2 x+37\right), & \dot{x}=v\left(x\left(-\frac{3 y}{b_{2}}-2\right)+w\right), \\
\dot{y}=y\left(\frac{v\left(\frac{8214 w}{w+x+1}+3555 x-6475\right)}{11850 b_{2}}-\frac{40 z}{b_{2}+y}\right), & \dot{z}=\frac{10 z\left(y-b_{2}\right)}{b_{2}+y} .
\end{array}
$$

Therefore, the equilibrium point is $\mathrm{P}_{0}=\left(\frac{175}{12}, \frac{35}{12}, \mathrm{~b}_{2}, \frac{7 \mathrm{v}}{160}\right)$ and $\mathrm{b}_{20}=\frac{1}{10}\left(\frac{28749}{37756}+\frac{139915489455}{8389718423 \mathrm{v}}\right)$. Taking $v=110$ we have that $b_{20} \approx 0.0913051$ and $P_{0}$ is $\left(14.5833,2.91667, b_{2}, 4.8125\right)$. The first Lyapunov coefficient is $\ell_{1}\left(\mathrm{P}_{0}, \mathrm{~b}_{20}\right) \approx-0.00118615$. Hence the system exhibits a supercritical Hopf bifurcation at $\mathrm{P}_{0}$ with respect to $b_{2}$. Setting $b_{2}=b_{20}-10^{-3} \approx 0.0903051$, we have in Figure 3 a projection to the $(x, y, z)$ space of an orbit with initial condition $\mathrm{Q}_{0}=(14.5933,2.92667,0.100305,4.8225)$ which tends to the stable limit cycle. Figure 4 shows the corresponding time series.

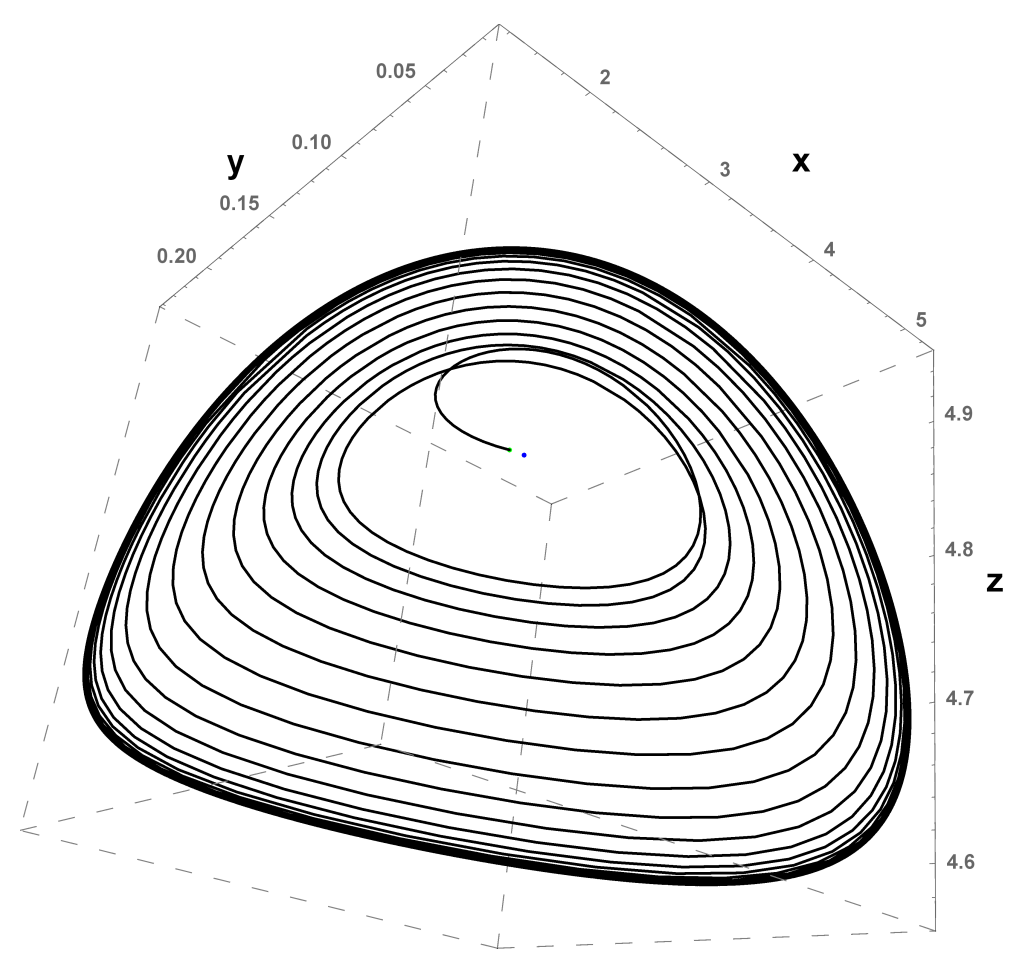

Figure 3: Projection of limit cycle (one equilibrium, $f_{1}$ Holling II).

Remark 5.2. The bifurcation parameter value $b_{20}$ depends directly on $v$ and is bounded below. Near the bifurcation value, the third coordinate of $\mathrm{P}_{0}$ (predator density) is approximately $\mathrm{b}_{20}$. The fourth coordinate of $\mathrm{P}_{0}$ (superpredator density) is directly proportional to $v$. 



Figure 4: Time series (One equilibrium, $f_{1}$ Holling II).

Example 5.3. From the hypothesis in Theorem 3.7 the differential system (1.1) takes the form

$$
\begin{aligned}
\dot{w} & =\frac{365 d_{3} w}{1131}\left(-\frac{1560 w_{0} y}{13 w y_{0}+4 w_{0} y_{0}+13 x y_{0}}-\frac{169(w+x)}{2 w_{0}}+221\right), \\
\dot{x} & =\frac{365 d_{3}\left(9 w y_{0}-x\left(y+8 y_{0}\right)\right)}{87 y_{0}}, \\
\dot{y} & =\frac{d_{3} y}{3828}\left(\frac{133225\left(44 b_{2} w+y_{0}\left(31 w-4 w_{0}-13 x\right)\right)}{\left(13 w+4 w_{0}+13 x\right) y_{0}}-\frac{3828 z\left(b_{2}+y_{0}\right)}{y_{0} c_{3}\left(b_{2}+y\right)}+\frac{133225 x}{w_{0}}\right), \\
\dot{z} & =\frac{b_{2} d_{3} z\left(y-y_{0}\right)}{y_{0}\left(b_{2}+y\right)} .
\end{aligned}
$$

Therefore, $\mathrm{b}_{20}=\frac{2361971095625 \mathrm{y}_{0}}{131632971184}$

$$
P_{0}=\left(w_{0}, w_{0}, y_{0}, \frac{26645}{522} c_{3}\left(b_{2}+y_{0}\right)\right) \text { and } P_{1}=\left(\frac{2 w_{0}}{13}, \frac{2 w_{0}}{13}, y_{0}, \frac{133225 b_{2} c_{3}}{4524}\right)
$$

Taking $c_{3}=\frac{1}{2}, d_{3}=1, w_{0}=58$ and $y_{0}=1$ we have that $b_{20} \approx 17.9436$ and the first Lyapunov coefficient at $\mathrm{P}_{0}$ is $\ell_{1}\left(\mathrm{P}_{0}, \mathrm{~b}_{20}\right) \approx-0.0000736782$. Hence the system exhibits a supercritical Hopf bifurcation at $\mathrm{P}_{0}$ with respect to $b_{2}$. Setting $b_{2}=b_{20}-10^{-1} \approx 17.8436$, we have in Figure 5 a projection to the $(x, y, z)$ space of an orbit with initial condition $\mathrm{Q}_{0}=(58.01,58.01,1.01,480.937)$ which tends to the stable limit cycle. Figure 6 shows the corresponding time series. 


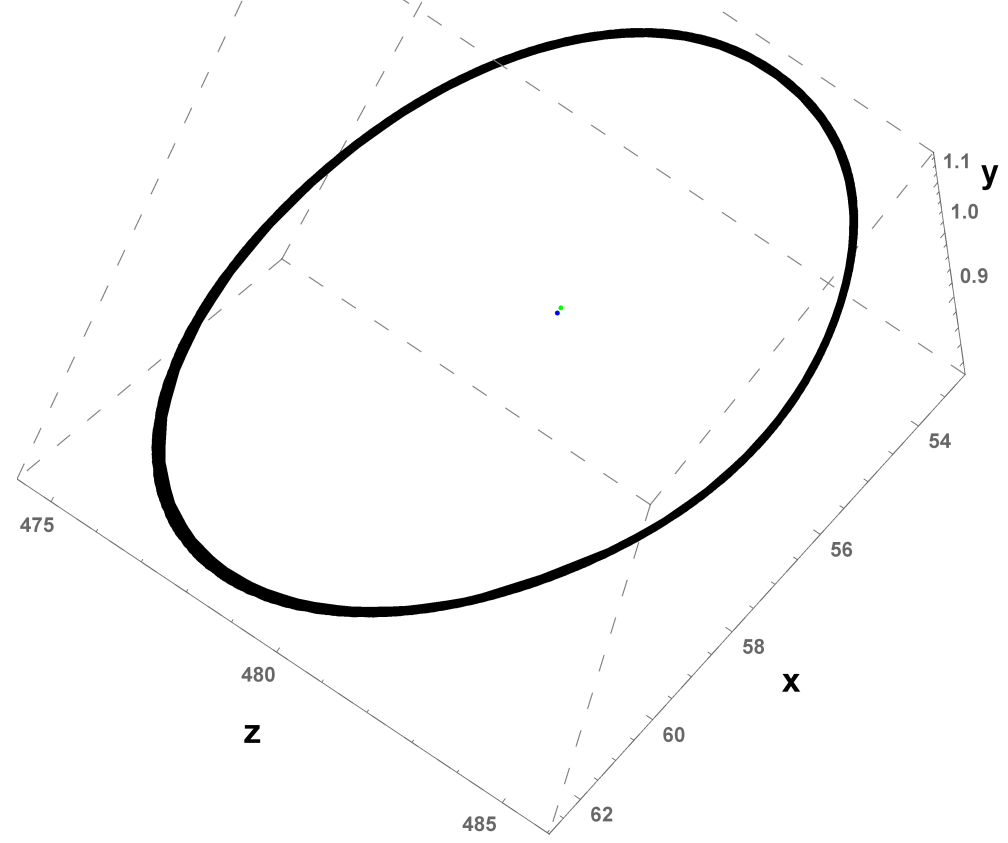

Figure 5: Projection of limit cycle with respect to $P_{0}$ (two equilibria, $f_{1}$ Holling II).
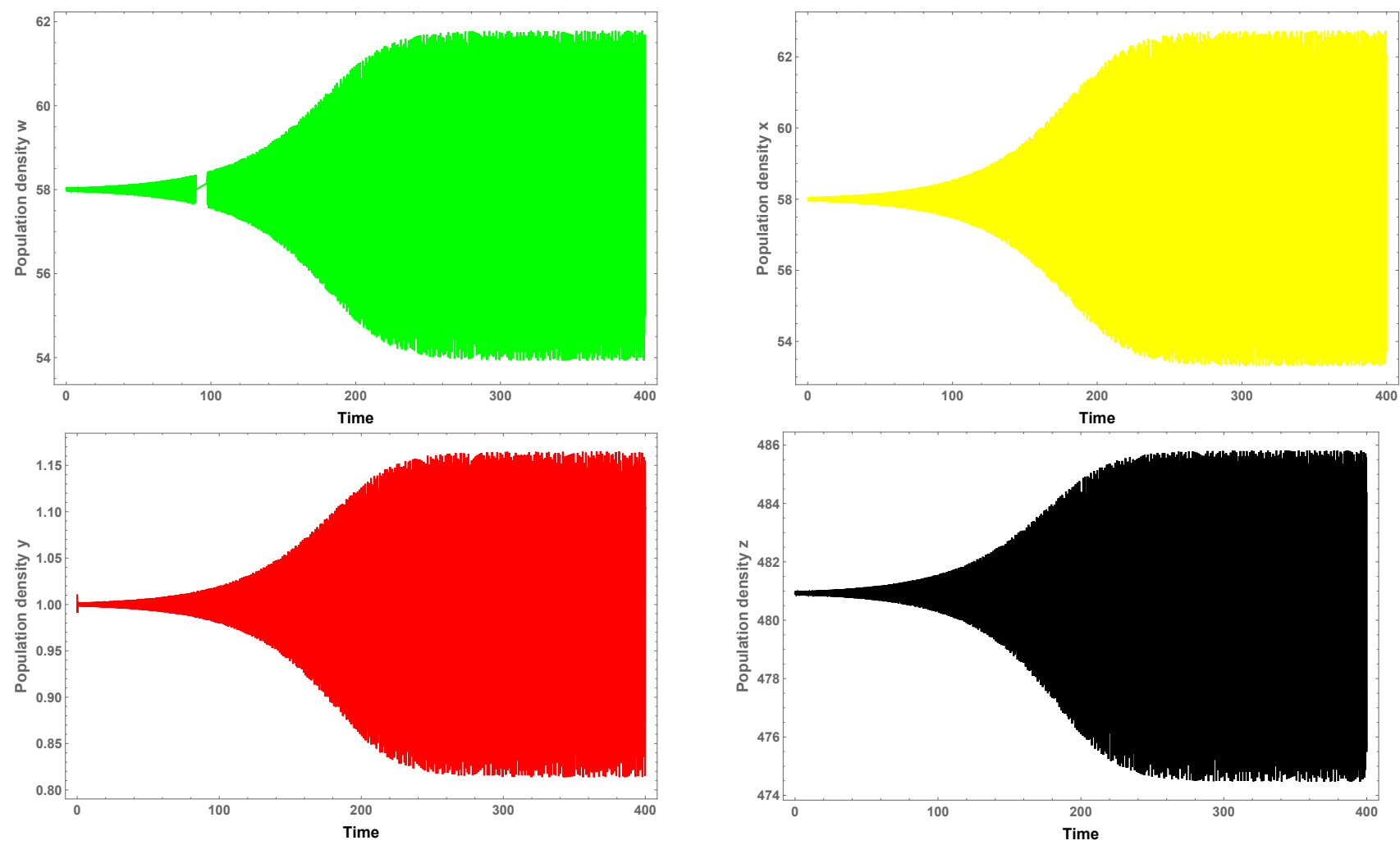

Figure 6: Time series with respect to $P_{0}$ (two equilibria, $f_{1}$ Holling II).

Remark 5.4. When the system (1.1) has two equilibrium points and the parameter $b_{2}$ is near to the bifurcation value $b_{20}$, the third and fourth coordinates of $P_{0}$ are directly proportional to $y_{0}$. 


\section{2. $\mathrm{f}_{1}$ Holling $I V$}

In the following three examples the functional response $f_{1}$ in the system (1.1) is Holling type IV.

Example 5.5. From the hypothesis in Theorem 4.4 the differential system (1.1) takes the form

$$
\begin{array}{ll}
\dot{w}=\frac{529}{800} v w\left(-\frac{8 y}{\mathrm{~b}_{2}\left(w^{2}+x+2\right)}-w-x+4\right), & \dot{x}=\frac{1}{2} v\left(2 w-\frac{x\left(\mathrm{~b}_{2}+\mathrm{y}\right)}{\mathrm{b}_{2}}\right), \\
\dot{y}=\frac{1}{400} \mathrm{y}\left(v\left(529\left(\frac{4 w}{w^{2}+x+2}-1\right)+100 x\right)-\frac{800 \mathrm{~d}_{3} z}{\mathrm{c}_{3}\left(\mathrm{~b}_{2}+\mathrm{y}\right)}\right), & \dot{z}=\frac{\mathrm{d}_{3} z\left(\mathrm{y}-\mathrm{b}_{2}\right)}{\mathrm{b}_{2}+\mathrm{y}} .
\end{array}
$$

Therefore, if $v=110, b_{2}=\frac{1}{2}, c_{3}=\frac{1}{2}$, then $d_{31}=\frac{29868927}{32000} \approx 933.404$ and the positive equilibrium point is $P_{0}=\left(1,1, \frac{1}{2}, \frac{55}{8 d_{3}}\right)$.

The first Lyapunov coefficient is $\ell_{1}\left(\mathrm{P}_{0}, \mathrm{~d}_{31}\right) \approx-0.222841$. Hence the system exhibits a supercritical Hopf bifurcation at $P_{0}$ with respect to $d_{3}$. Setting $d_{3}=b_{31}+10^{-1} \approx 933.504$, we have in Figure 7 a projection to the $(x, y, z)$ space of an orbit with initial condition $Q_{0}=(1.00001,1.00001,0.50001,0.00737472)$ which tends to the stable limit cycle. Figure 8 shows the corresponding time series.

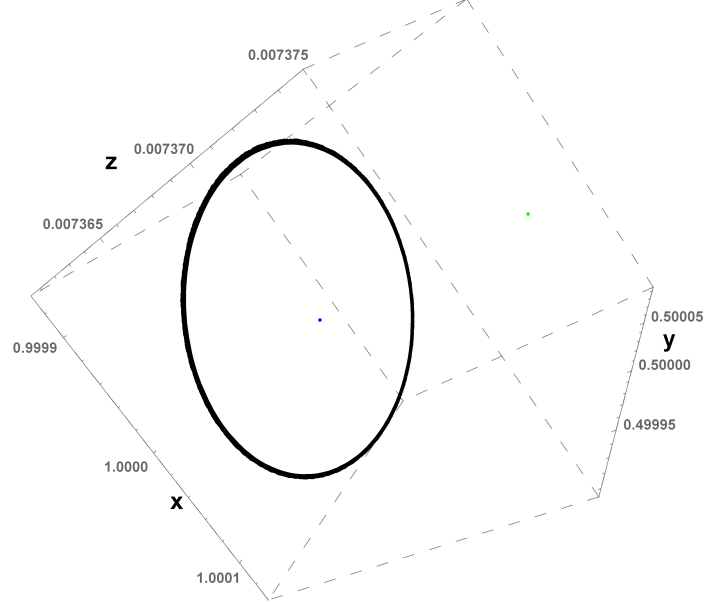

Figure 7: Projection of limit cycle with respect to $P_{0}$ (one equilibrium, $f_{1}$ Holling IV).

Example 5.6. From the hypothesis in Theorem 4.7 the differential system (1.1) takes the form

$$
\begin{array}{ll}
\dot{w}=\frac{29445 \mathrm{~d}_{3} w}{246208}\left(-\frac{3125 y}{y_{0}\left(w^{2}+x+\frac{241}{64}\right)}-128 w-128 x+832\right), & \dot{x}=\frac{29445 d_{3}\left(3 w y_{0}-x\left(2 y+y_{0}\right)\right)}{3847 y_{0}}, \\
\dot{y}=\frac{d_{3} y\left(b_{2}+y_{0}\right)}{984832 y_{0}}\left(-\frac{984832 z}{c_{3}\left(b_{2}+y\right)}+\frac{51675975 w}{w^{2}+x+\frac{241}{64}}+876096 x-1752192\right), & \dot{z}=\frac{b_{2} d_{3} z\left(y-y_{0}\right)}{y_{0}\left(b_{2}+y\right)}
\end{array}
$$

Therefore, $\mathrm{b}_{20}=\frac{95480385736 \mathrm{y}_{0}}{16391886457}$ and

$$
P_{0}=\left(2,2, y_{0}, \frac{2067039 c_{3}\left(b_{2}+y_{0}\right)}{192350}\right), P_{1}=\left(\frac{1}{8}, \frac{1}{8}, y_{0}, \frac{13689 c_{3}\left(b_{2}+y_{0}\right)}{1231040}\right) .
$$

Taking $y_{0}=1, d_{3}=1, c_{3}=\frac{1}{2}$, we have that $b_{20} \approx 5.82486$ and the first Lyapunov coefficient is $\ell_{1}\left(P_{0}, b_{20}\right) \approx$ -1.91318 . Hence the system exhibits a supercritical Hopf bifurcation at $P_{0}$ with respect to $b_{2}$. Setting $b_{2}=$ $b_{20}-10^{-1} \approx 5.72486$, we have in Figure 9 a projection to the $(x, y, z)$ space of an orbit with initial condition $\mathrm{Q}_{0}=(2.01,2.01,1.01,36.1435)$ which tends to the stable limit cycle. Figure 10 shows the corresponding time series. 



Figure 8: Time series with respect to $\mathrm{P}_{0}$ (one equilibrium, $\mathrm{f}_{1}$ Holling IV).

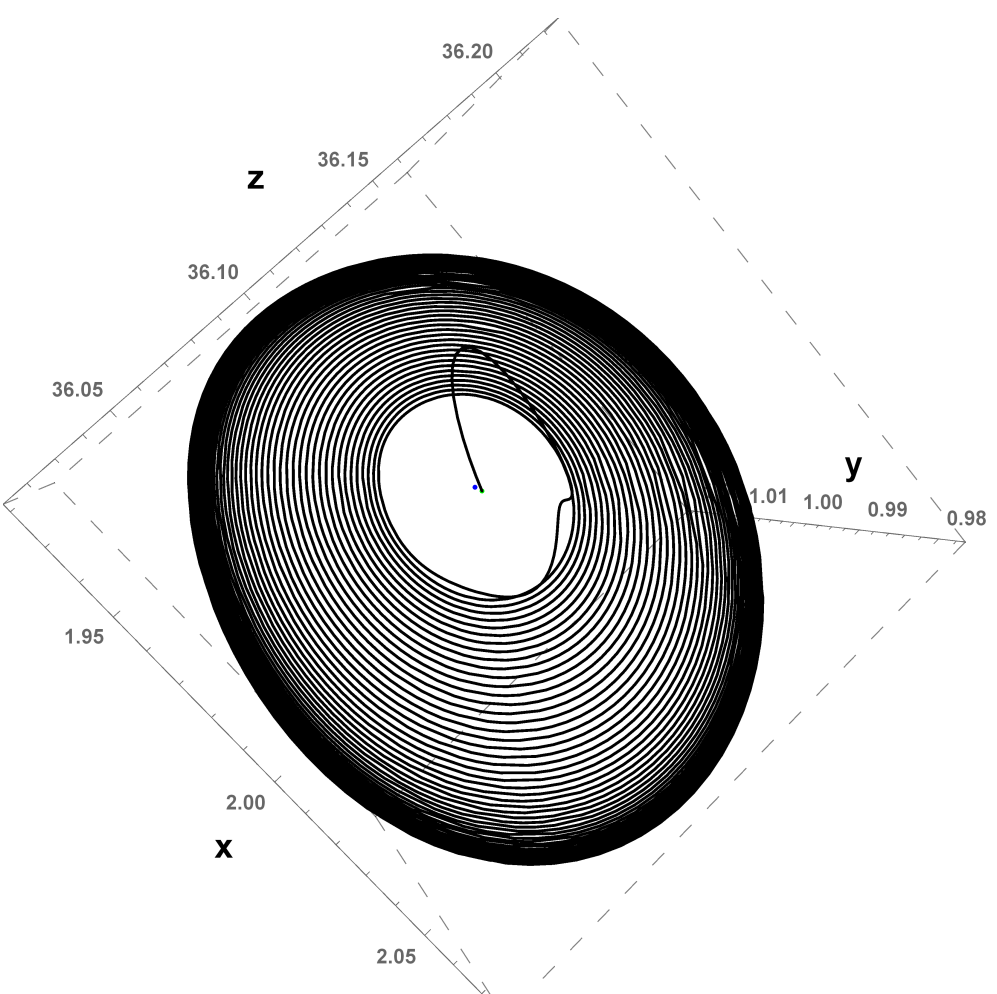

Figure 9: Projection of limit cycle with respect to $P_{0}$ (two equilibria, $f_{1}$ Holling IV).

Remark 5.7. When the system (1.1) has two equilibrium points and the parameter $b_{2}$ is near to the bifurcation value $b_{20}$, the third and fourth coordinates of $P_{0}$ are directly proportional to $y_{0}$. 

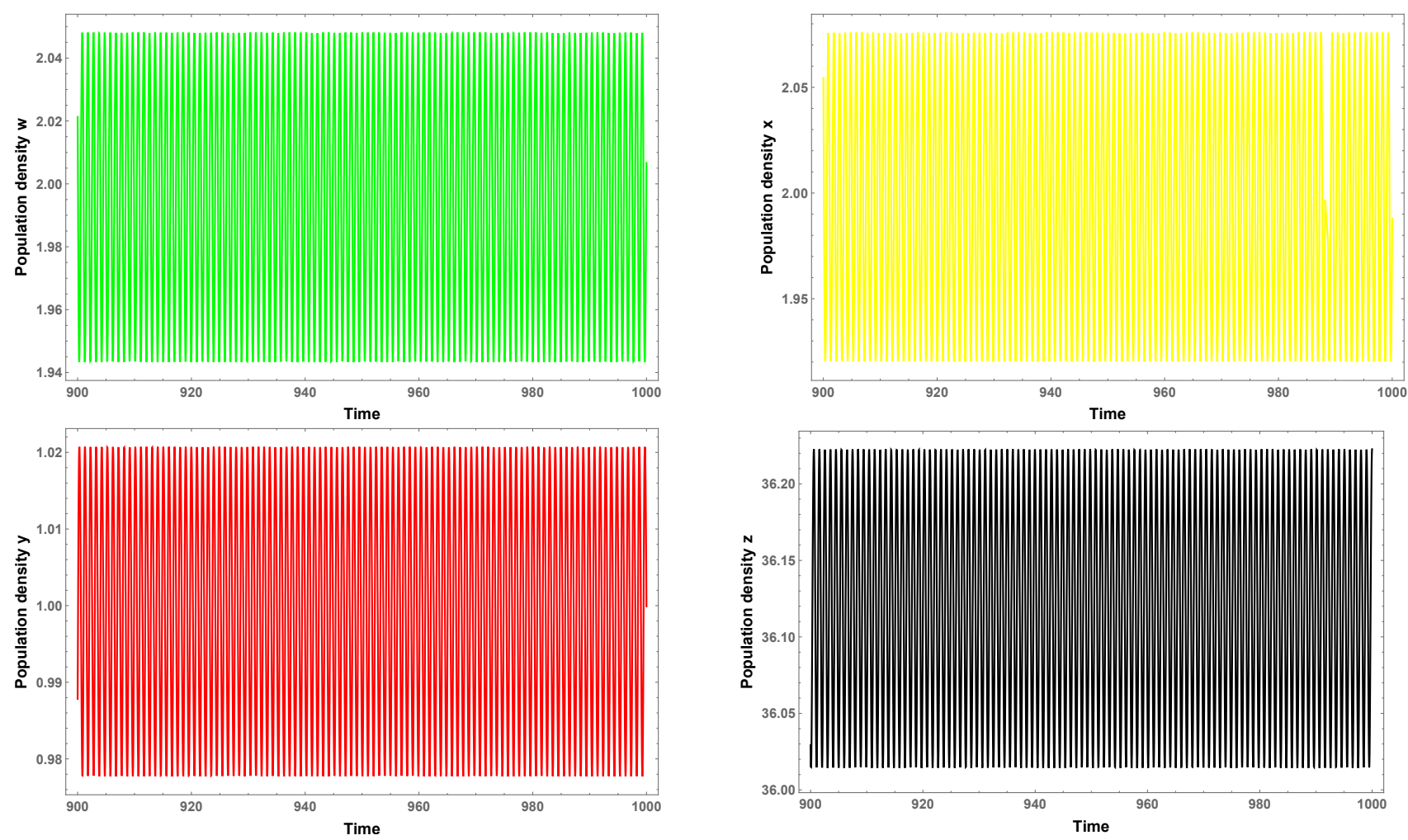

Figure 10: Time series with respect to $P_{0}$ (two equilibria, $f_{1}$ Holling IV).

Example 5.8. From the hypothesis in Theorem 4.10 i) the differential system (1.1) takes the form

$$
\begin{aligned}
\dot{w} & =\frac{21873 d_{3} w}{45928192}\left(-\frac{37485 y}{y_{0}\left(w^{2}+x+\frac{963}{64}\right)}-128 w-128 x+2496\right), \\
\dot{x} & =\frac{65619 d_{3}\left(6 w y_{0}-x\left(2 y+y_{0}\right)\right)}{1435256 y_{0}}, \\
\dot{y} & =\frac{d_{3} y\left(b_{2}+y_{0}\right)}{180038512640 y_{0}}\left(256\left(-\frac{703275440 z}{c_{3}\left(b_{2}+y\right)}-100489\right)+\frac{3057779781 w}{w^{2}+x+\frac{963}{64}}+3215648 x\right), \\
\dot{z} & =\frac{b_{2} d_{3} z\left(y-y_{0}\right)}{y_{0}\left(b_{2}+y\right)} .
\end{aligned}
$$

We have that $b_{20}=\frac{13416348622408722 y_{0}}{582645121928723}$ and the positive equilibrium points are

$$
P_{0}=\left(4,8, y_{0}, \frac{20801223\left(b_{2}+y_{0}\right)}{23911364960}\right), P_{1}=\left(\frac{1}{8}, \frac{1}{4}, y_{0}, \frac{100489\left(b_{2}+y_{0}\right)}{900192563200}\right), P_{2}=\left(\frac{3}{8}, \frac{3}{4}, y_{0}, \frac{2066958241\left(b_{2}+y_{0}\right)}{15303273574400}\right) .
$$

Taking $d_{3}=1, c_{3}=\frac{1}{2}$ and $y_{0}=40$, the first Lyapunov coefficient is $\ell_{1}\left(P_{0}, b_{20}\right) \approx-0.0134592$. Hence the system exhibits a supercritical Hopf bifurcation at $P_{0}$ with respect to $b_{2}$ and $P_{1}$ is locally asymptotically stable. Setting $b_{2}=b_{20}-10^{-2} \approx 921.055$, we have in Figure 11 a projection to the $(x, y, z)$ space of an orbit with initial condition $\mathrm{Q}_{0}=(4.01,8.01,40.01,0.846051)$ which tends to the stable limit cycle. Figure 12 shows the corresponding time series.

Remark 5.9. When the system (1.1) has three equilibrium points and the parameter $b_{2}$ is near to the bifurcation value $b_{20}$, the third and fourth coordinates of $P_{0}$ are directly proportional to $y_{0}$. 

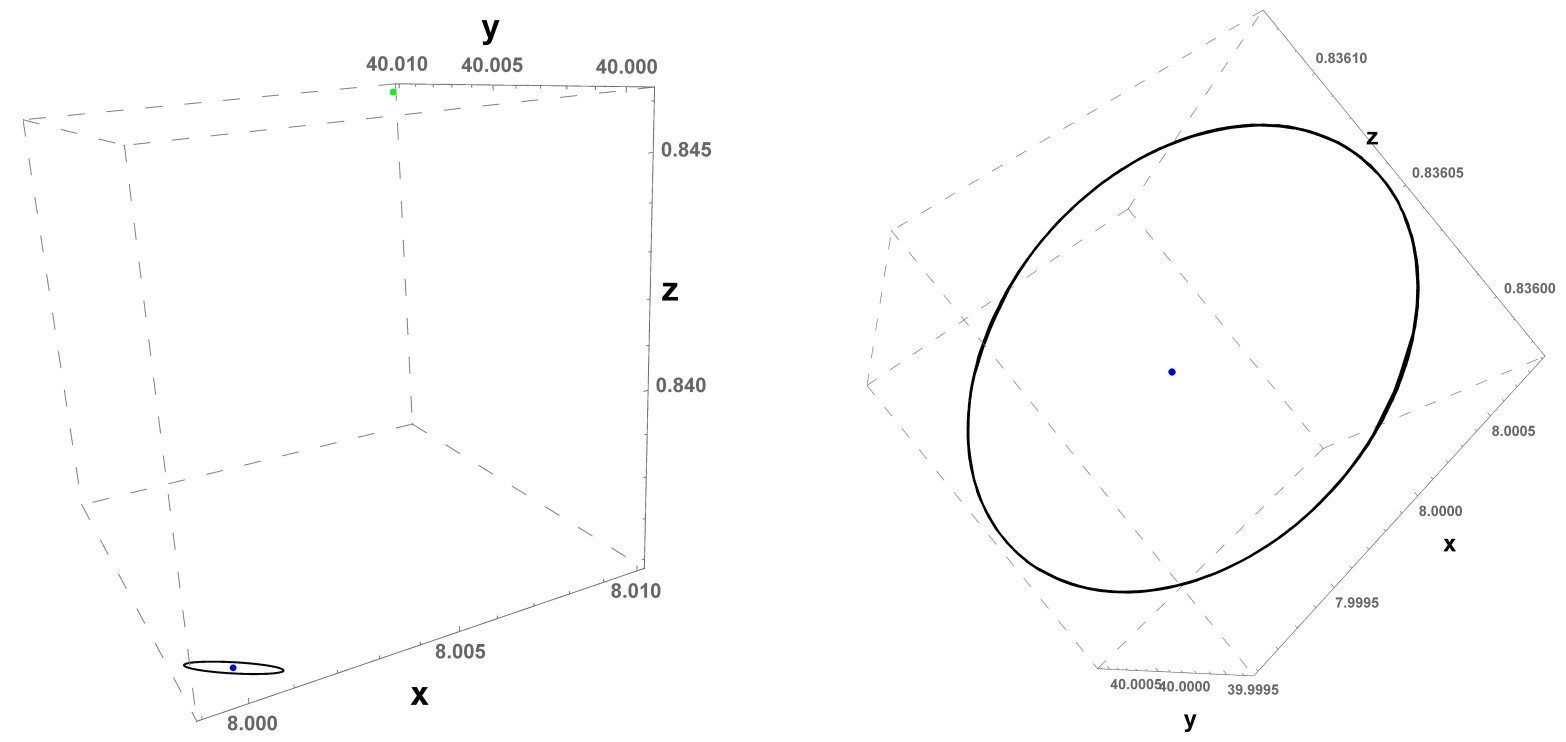

Figure 11: Projection of limit cycle with respect to $P_{0}$ (three equilibria, $f_{1}$ Holling IV).
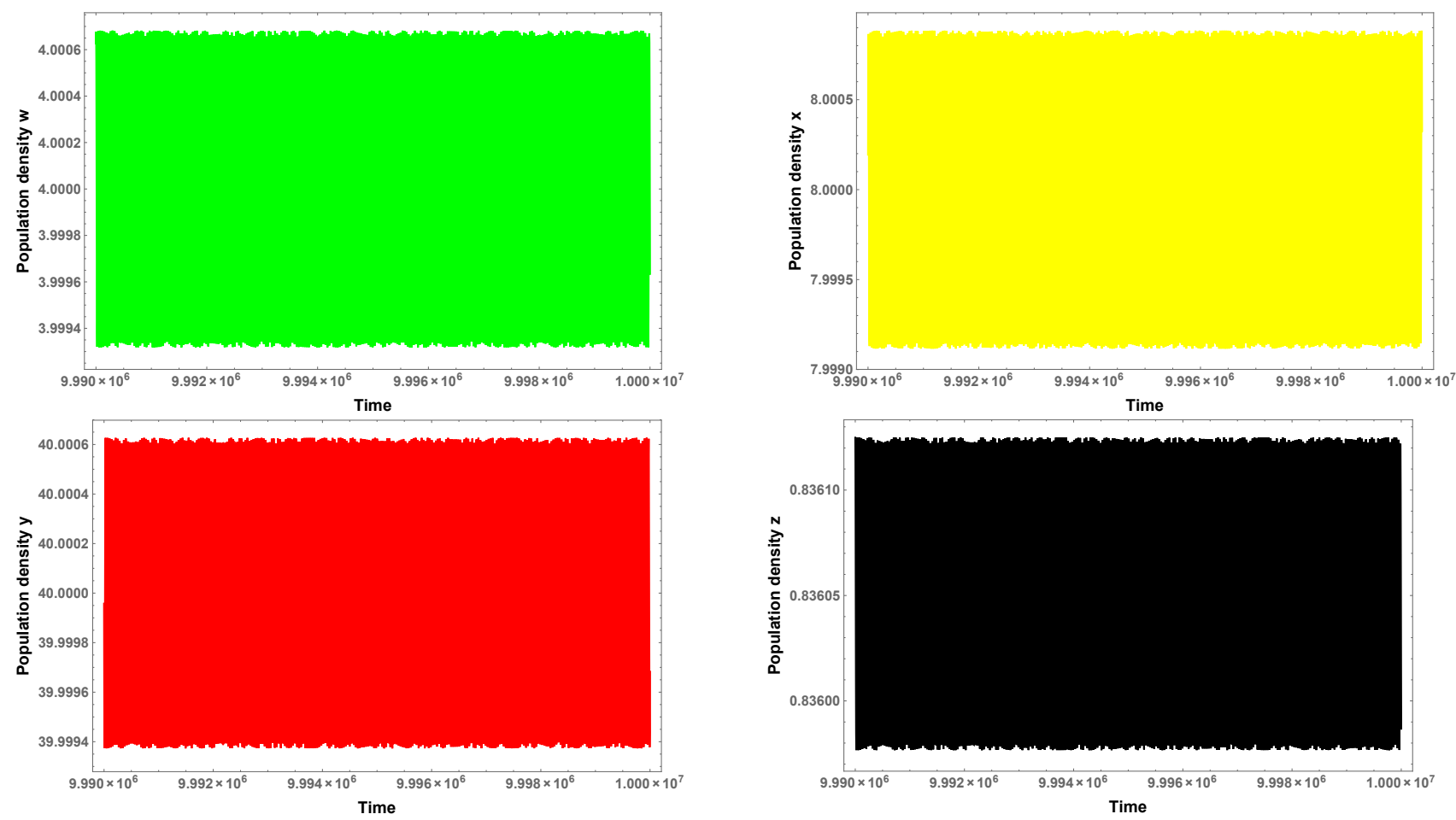

Figure 12: Time series with respect to $P_{0}$ (three equilibria, $f_{1}$ Holling IV).

\section{Conclusions}

The dynamics of the tritrophic chain model with age structure in the prey given by the differential sytem (1.1) is determined under sufficient conditions on the parameter space. We considered two different types of interaction between predator and reproductive population prey: through a Holling type II or IV functional response $f_{1}$.

If $f_{1}$ is Holling type II it is possible to have one or two positive equilibria $P_{0}$ and $P_{1}$. There are parameters conditions such that the differential system (1.1) has a Hopf bifurcation at $\mathrm{P}_{0}$ with respect to the parameter $b_{2}$ representing the handling time. If there is only one equilibrium point, the bifurcation could be sub- or super-critical, whereas if there are two equilibria, it is only supercritical and the the other 
equilibrium point is locally unstable.

When $f_{1}$ is Holling type IV, there are sufficient conditions to have one, two or three positive equilibrium points, $\mathrm{P}_{0}, \mathrm{P}_{1}, \mathrm{P}_{2}$. In all cases the differential system (1.1) exhibits a supercritical Hopf bifurcation at $\mathrm{P}_{0}$. In the first case, the bifurcation is with respect to $d_{3}$ which represents the mortality superpredator rate growth. In the second case, the bifurcation is with respect to $b_{2}$ and $P_{1}$ is locally unstable. In the third case, there is a non simultaneous Hopf bifurcation at $P_{0}$ and $P_{1}$ with respect to $b_{2}$, and $P_{2}$ is locally unstable. From Theorems 3.4, 3.7, 4.7, and 4.10 we have that given a predator density there are parameters values that guarantee the coexistence coming from a supercritical Hopf bifurcation whose bifurcation value is approximately the predator density. Finally, we emphasize that the differential system (1.1) may presents bistability (see Theorem 4.10 i)) when there is defense in the prey.

\section{References}

[1] J. R. Beddington, C. A. Free, Age structure effects in predator-prey interactions, Theoret. Population Biology, 9 (1976), 15-24. 1

[2] V. Castellanos, F. E. Castillo-Santos, M. A. Dela-Rosa, I. Loreto-Hernández, Hopf and Bautin Bifurcation in a Tritrophic Food Chain Model with Holling Functional Response Types III and IV, Internat. J. Bifur. Chaos Appl. Sci. Engrg., 28 (2018), 24 pages. 3.1

[3] J. M. Cushing, M. Saleem, A predator prey model with age structure, J. Math. Biol., 14 (1982), 231-250. 1

[4] J. H. P. Dawes, M. O. Souza, A derivation of Holling's type I, II and III functional responses in predator prey systems, J. Theoret. Biol., 327 (2013), 11-22. 1

[5] M. Falconi, The effect of the prey age structure on a predator-prey system, Sci. Math. Jpn., 64 (2006), 267-275. 1

[6] A. Hastings, D. Wollkind, Age structure in predator-prey systems. I. A general model and a specific example, Theoret. Population Biol., 21 (1982), 44-56.

[7] Y. A. Kuznetsov, Elements of applied Bifurcation Theory, Springer-Verlag, New York, (2004).

[8] Y. A. Kuznetsov, Andronov-Hopf bifurcation, Scholarpedia, 1 (2006), 6 pages. 1

[9] E. D. LeCaven, C. Kipling, J. C. McCormack, A study of the numbers, biomass and year class strengths of pereh (Perca fluviatillis L) in Winderemere from 1941-1966, J. Anim. Ecol., 46 (1977), 281-307. 2, 3.1 3.1, 3.1 1

[10] M. LLoyd, H. S. Dybas, The periodical cicada problem. I. Population ecology, Evolution, 20 (1966), 133-149.

[11] M. LLoyd, H. S. Dybas, The periodical cicada problem. II. Evolution, Evolution, 20 (1966), 466-505. 1

[12] J. E. Marsden, M. McCracken, The Hopf Bifurcation and Its Applications, Springer-Verlag, New York, (1976). 3.1

[13] J. D. Murray, Mathematical biology. I: An introduction, Springer, New York, (2004). 1

[14] L. Perko, Differential Equations and Dynamical Systems, Springer-Verlag, New York, (2001). 3.1

[15] J. Promrak, G. C. Wake, C. Rattanakul, Predator-prey model with age structure, ANZIAM J., 59 (2017), 155-166. 1

[16] R. Sigal, Algorithms for the Routh-Hurwitz Stability Test, Math. Comput. Modelling, 13 (1990), 69-77. 2

[17] G. T. Skalski, J. F. Gilliam, Functional responses with predator interference: Viable alternatives to the Holling type II model, Ecology, 82 (2001), 3083-3092. 1

[18] H. Tang, Z. Liu, Hopf bifurcation for a predator-prey model with agestructure, Appl. Math. Model., 40 (2016), $726-737$. 1

[19] D. J. A. Toth, Bifurcation structure of a chemostat model for an age-structured predator and its prey, J. Biol. Dyn., 2 (2008), 428-448. 1

[20] H. Y. Xi, L. H. Huang, Y. C. Qiao, H. Y. Li, C. X. Huang, Permanence and partial extinction in a delayed three-species food chain model with stage structure and time-varying coefficients, J. Nonlinear Sci. Appl., 10 (2017), 6177-6191. 1 